\title{
Maturation of a central brain flight circuit in Drosophila requires $\mathrm{Fz} 2 / \mathrm{Ca}^{2+}$ signaling
}

\author{
Tarjani Agrawal, Gaiti Hasan*
}

\author{
National Centre for Biological Sciences, Tata Institute of Fundamental Research, \\ Bangalore, India
}

\begin{abstract}
The final identity of a differentiated neuron is determined by multiple signaling events, including activity dependent calcium transients. Non-canonical Frizzled2 (Fz2) signaling generates calcium transients that determine neuronal polarity, neuronal migration, and synapse assembly in the developing vertebrate brain. Here, we demonstrate a requirement for $\mathrm{Fz} 2 / \mathrm{Ca}^{2+}$ signaling in determining the final differentiated state of a set of central brain dopaminergic neurons in Drosophila, referred to as the protocerebral anterior medial (PAM) cluster. Knockdown or inhibition of $\mathrm{Fz} 2 / \mathrm{Ca}^{2+}$ signaling during maturation of the flight circuit in pupae reduces Tyrosine Hydroxylase $(T H)$ expression in the PAM neurons and affects maintenance of flight. Thus, we demonstrate that $\mathrm{Fz} 2 / \mathrm{Ca}^{2+}$ transients during development serve as a pre-requisite for normal adult behavior. Our results support a neural mechanism where PAM neuron send projections to the $\alpha^{\prime}$ and $\beta^{\prime}$ lobes of a higher brain centre, the mushroom body, and function in dopaminergic re-inforcement of flight. DOI: 10.7554/eLife.07046.001
\end{abstract}

*For correspondence: gaiti@ ncbs.res.in

Competing interests: The authors declare that no competing interests exist.

Funding: See page 19

Received: 16 February 2015

Accepted: 07 May 2015

Published: 08 May 2015

Reviewing editor: Leslie C Griffith, Brandeis University, United States

Copyright Agrawal and Hasan. This article is distributed under the terms of the Creative Commons Attribution License, which permits unrestricted use and redistribution provided that the original author and source are credited.

\section{Introduction}

Genetically encoded developmental programs and neuronal activity together shape the neurotransmitter identity of developing neural circuits. In vertebrates, calcium transients generated by neuronal activity can influence neurotransmitter specification during development and in adults (Spitzer, 2012; Borodinsky et al., 2014). One mechanism of generating $\mathrm{Ca}^{2+}$ transients is non-canonical Wnt/Ca ${ }^{2+}$ signaling initiated by membrane bound Frizzled receptors and a trimeric G-protein (Slusarski et al., 1997). Such calcium signals are known to affect neuronal polarity, migration as well as synapse assembly in the developing and mature vertebrate brain (Varela-Nallar et al., 2010; Ciani et al., 2011). Wnt signaling was first identified in Drosophila where multiple genes encode Wnt and Fz proteins (van Amerongen and Nusse, 2009). However, the role of non-canonical Wnt/Ca ${ }^{2+}$ signaling during neural development and circuit maturation is poorly understood in invertebrates and its ability to stimulate $\mathrm{Ca}^{2+}$ transients during circuit maturation is unknown. In a screen for G-protein coupled receptors required for flight circuit maturation in Drosophila we identified dFrizzled2 (dFz2) and found that flight deficits upon $d F z 2$ knockdown can be suppressed by over-expression of the intracellular endoplasmic reticular $\mathrm{Ca}^{2+}$ sensor dSTIM (Agrawal et al., 2013). Adult neural circuits in Drosophila, including the flight circuit, form in the pupal stages (Consoulas et al., 2002), and it is known that maturation of the Drosophila flight circuit requires intracellular $\mathrm{Ca}^{2+}$ signaling (Banerjee et al., 2004; Agrawal et al., 2013). To understand the molecular and cellular basis for such flight deficits, we set out to map neurons that require $\mathrm{dFz} 2$ receptor signaling in the context of flight circuit maturation.

Insect flight requires computation of multiple sensory inputs and their integration with the flight motor system. This computation and integration presumably occurs in central neurons and allows for control of initiation, maintenance and cessation of voluntary flight bouts (Gotz, 1987; Strauss, 2002; Strausfeld and Hirth, 2013). Recent work has shown that central dopaminergic neurons in the ventral ganglion modulate a pair of direct flight muscle motor neurons required for wing coordination during flight initiation and cessation (Sadaf et al., 2015). In addition, central neurons that compute sensory 
eLife digest The fruit fly Drosophila melanogaster is an aerial acrobat. These insects can suddenly change direction in less than one hundredth of a second, explaining why a moving fly can be so difficult to swat. To perform their aerial manoeuvres, the flies continually combine information from multiple senses, including vision, hearing and smell, and use these data to control the activity of the neural circuits that support flight.

These flight circuits are established during the pupal stage of fly development, during which the fly transforms from a larva into its adult form. In 2013, researchers showed that a protein called dFrizzled2 must be present in pupae for flight circuits to mature correctly. This protein forms part of a pathway that ultimately controls which specific chemicals_called neurotransmitters-are released by neurons to communicate with other cells. Agrawal and Hasan-who worked on the 2013 study-now extend their findings to investigate the role of dFrizzled2 in more detail.

The new experiments show that for the flight circuits to mature, dFrizzled2 must be active in a cluster of neurons known collectively as PAM. Specifically, dFrizzled2 is needed to make an enzyme that helps to produce a neurotransmitter called dopamine. This in turn enables the PAM neurons to communicate with a region of the fruit fly brain called the mushroom body, which it thought to play an important role in complex behaviors such as reward-based learning.

The absence of dFrizzled2 results in adult flies that rarely remain airborne for more than $20 \mathrm{~s}$ at a time, whereas normal flies can typically fly for over $700 \mathrm{~s}$. Given that dopamine is known to signal reward, one possibility is that the dopamine signals from the PAM neurons to the mushroom body serve as a reward to encourage continuous flight. Mutant flies that lack dFrizzled2-and thus these dopamine signals-lose their motivation to fly after only a few seconds.

Overall, Agrawal and Hasan's findings suggest that the mushroom body has an important role in coordinating a fly's movements with information from it senses. Future research will be needed to determine exactly how the mushroom body performs this role.

DOI: 10.7554/eLife.07046.002

information in real time and control the timing of a flight bout must exist but remain unknown. Most complex insect behaviors, including flight, are modulated by various monoamines and neuropeptides and in Drosophila, flight can be modulated by octopamine, serotonin and dopamine as well as several neuropeptides (Taghert and Nitabach, 2012; Sadaf and Hasan, 2014; Van Breugel et alo, 2014). Here, we show for the first time that dFz2 signaling drives the expression of Tyrosine Hydroxylase $(\mathrm{TH})$, the rate-limiting enzyme in dopamine synthesis (Friggi-Grelin et al., 2003), during circuit maturation, in a specific set of central brain dopaminergic neurons, called the protocerebral anterior medial (PAM) neurons. The PAM cluster consists of approximately 90 dopaminergic neurons, which project to different regions of a higher brain structure called the mushroom body (MB). PAM-MB connectivity has been studied for its role in olfactory associative learning and memory (Aso et al., 2012; Liu et al., 2012) where it is thought to signal reward reinforcement. More recently, a PAM-MB circuit was shown to control negative geotaxis behavior in flies (Riemensperger et al., 2013). Our studies demonstrate the presence of a novel PAM-MB flight circuit and support a role for PAM-MB synapses in dopaminergic re-inforcement of flight bouts.

\section{Results}

\section{Flight deficits in adult Drosophila arise from reduced $\mathrm{dFz2}$ function in pupal dopaminergic neurons}

To identify neurons, which require dFz2 function for flight, an RNAi strain ( $d F z 2-I R$ ) was expressed in independent neurotransmitter domains with the help of the UASGAL4 system for cell and tissue specific expression (Brand and Perrimon, 1993). Amongst the neuronal domains tested, significant flight deficits were observed upon knockdown of $d F z 2$ in aminergic neurons (60\% flight time; DdcGAL4) and in dopaminergic neurons (45\% flight time; THGAL4) (Figure 1A, Video 1). Because, DdcGAL4 drives expression in serotonergic and dopaminergic neurons, we tested flies with knockdown of dFz2 in serotonergic neurons (TRHGAL4). These flies exhibit normal flight bouts in the tethered flight assay (Figure 1A). Similarly, normal flight bouts were observed in flies with 


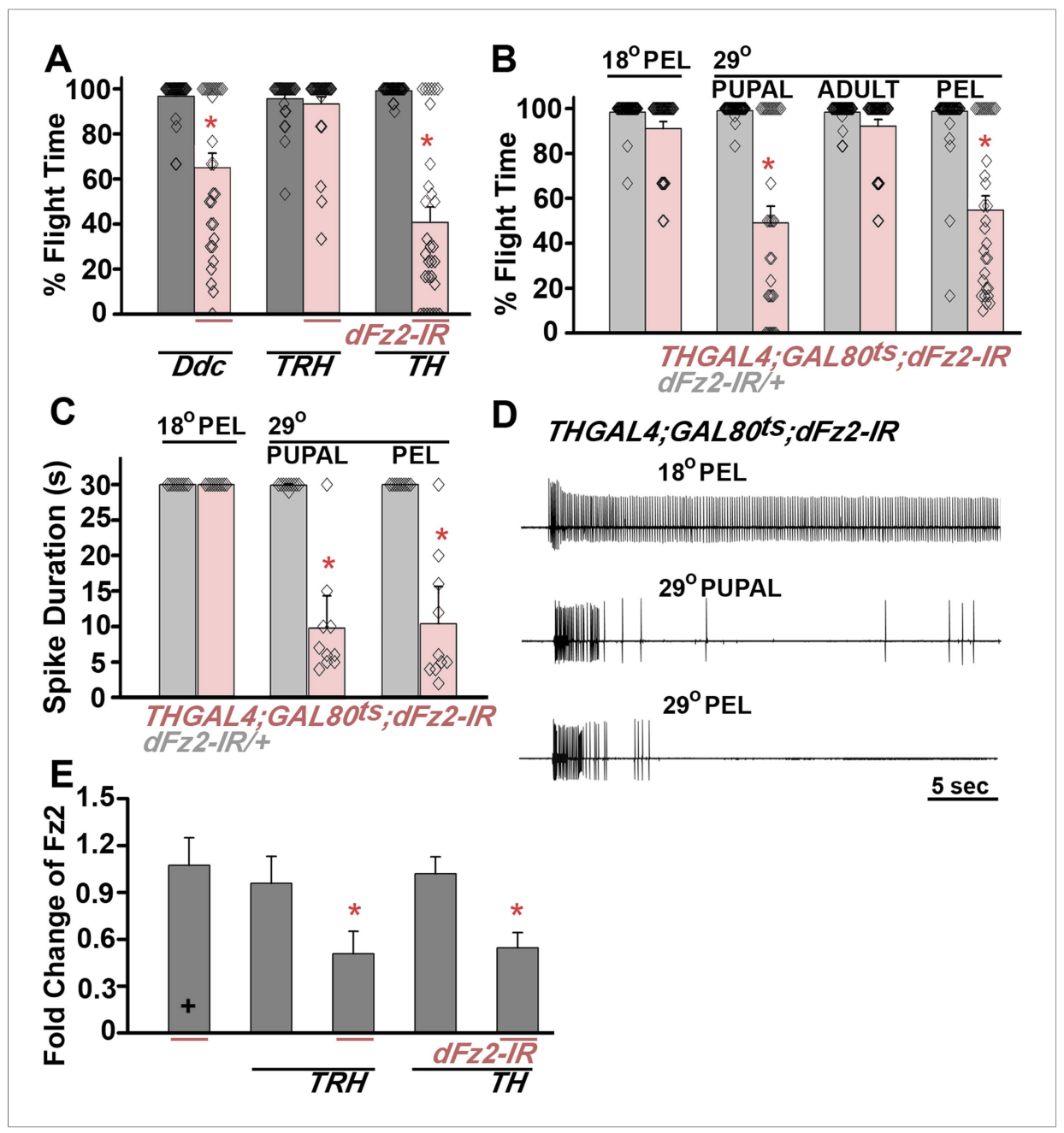

Figure 1. dFz2 function is required in dopaminergic neurons during development for normal adult flight. (A) Percentage flight times of individuals after knockdown of $d F z 2$ in aminergic neurons (DdcGAL4), serotonergic neurons (TRHGAL4), dopaminergic neurons (THGAL4). Knockdown of dFz2 in aminergic neurons (DdcGAL4, first bar in red) and in dopaminergic neurons (THGAL4, third bar in red) showed reduced flight. Knockdowns were compared to their respective GAL4 controls (gray bars; ${ }^{*} p<0.001$, Mann-Whitney U-test). (B) Percentage flight times of $d F z 2-I R$ heterozygotes (gray bars) and flies with knockdown of $d F z 2$ in dopaminergic neurons (red bars) at specific developmental stages by temperature controlled THGAL4; GAL80 ${ }^{\text {ts }}$ expression are shown. Flies with knockdown during pupal development exhibit reduced flight similar to knockdown post-egg laying (PEL) as compared to controls ( ${ }^{*} p<0.001$, Mann-Whitney U-test). (C) Durations of rhythmic action potentials recorded from the DLMs of air-puff stimulated tethered flies. Bars represent the mean spike duration and diamonds represent the spike duration of an individual recording ( ${ }^{*} p<0.001$, Mann-Whitney U-test). (D) Representative traces of electrophysiological recordings from DLMs of individuals with $d F z 2$ knockdown at the indicated developmental stages are shown. (E) Quantification of $d F z 2$ transcript levels after knockdown by $d F z 2$ RNAi in serotonergic (TRHGAL4) and dopaminergic (THGAL4) neurons. The Y-axis represents log2 fold changes calculated by the $\Delta \Delta \mathrm{Ct}$ method. Each value is the mean \pm SEM of three independent experiments, obtained from three independent RNA samples ( ${ }^{*} p<0.05$, one-way ANOVA).

DOI: $10.7554 /$ LLife. 07046.003

The following figure supplements are available for figure 1:

Figure supplement 1. Normal flight in flies with knockdown of dFz2 in non-dopaminergic neurons. DOI: 10.7554/eLife.07046.004

Figure supplement 2. Expression of multiple dFz2-IR strains in dopaminergic neurons exhibits flight defects. DOI: 10.7554/eLife.07046.005 


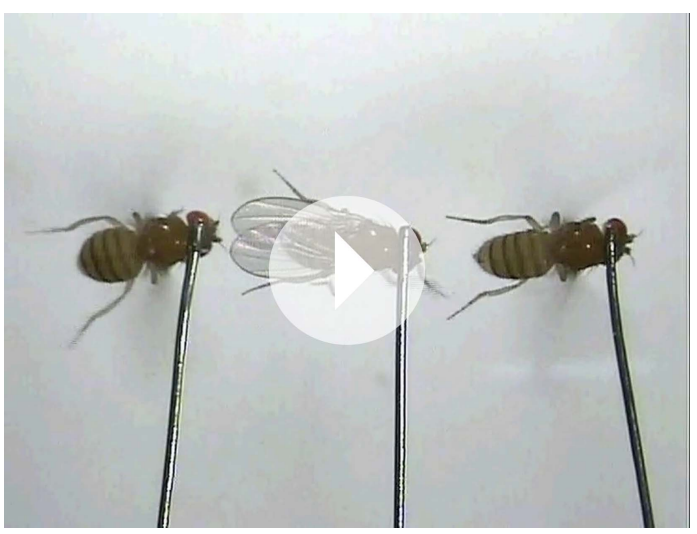

Video 1. dFz2 knockdown in dopaminergic neurons result in flight defect. Real time video recording of airpuff induced flight in the following genotypes from left to right. (1) THGAL4/+, (2) THGAL4;dFz2-IR, (3) $d F z 2-I R /+$. Following a gentle air-puff THGAL4; $\mathrm{dFz} 2-I R$ flies were able to initiate but not maintain flight for as long as control flies of the genotypes THGAL4/+ and $d F z 2-I R /+$ DOI: 10.7554/eLife.07046.006

flight circuit (Fernandes and Vijayaghova, 1993 ; Consoulas et al., 2002). We therefore determined the developmental stage at which dFz2 function is required in dopaminergic neurons for flight. For this purpose, we used the TARGET (temporal and regional gene expression targeting) system (McGuire et al., 2003). TARGET regulates GAL4 expression by a temperature sensitive GAL80 ${ }^{\text {ts }}$ element, which can be expressed and repressed at $18^{\circ} \mathrm{C}$ and $30^{\circ} \mathrm{C}$, respectively (McGuire et al., 2004). Experimental animals of the genotype THGAL4, $G A L 80^{\text {ts }} ; d F z 2-I R$ were shifted to the permissive GAL4 expression temperature $\left(30^{\circ} \mathrm{C}\right)$ either during pupal or adult stages. This allowed stage-specific knockdown of $d F z 2$. Upon knockdown of $d F z 2$ in $\mathrm{TH}$-expressing neurons through pupal development a significant reduction of flight time was observed, whereas normal flight bouts, as measured for $30 \mathrm{~s}$, were observed upon knockdown of $d F z 2$ in adults (Figure 1B). Flight deficits upon knockdown of $d F z 2$ in pupae were equivalent to those observed upon knockdown throughout post-embryonic development confirming that $\mathrm{dFz} 2 \mathrm{re}-$ quirement for flight is primarily in pupal dopaminergic neurons during circuit maturation. A physiological correlate of flight is rhythmic patterns of action potentials recorded from the dorsal longitudinal muscles (DLMs) during tethered flight. A reduction in duration of flight patterns was observed upon knockdown of $d F z 2$ in $T H$-expressing neurons during pupal development (Figure 1C,D). $d F z 2$ knockdown at pupal and adult stages in TH-expressing neurons was confirmed by qPCR. As a control, we also confirmed $d F z 2$ knockdown in serotonergic neurons targeted by TRHGAL4 where no flight deficits were observed (Figure 1E). The specificity of flight deficits obtained upon $d F z 2$ knockdown was tested by expression of three additional RNAi strains for dFz2 (BL27568, BL31390, and BL31312). Significant flight defects ranging from $67 \%$ to $72 \%$ were obtained upon knockdown through post-embryonic development (Figure 1-figure supplement 2). The difference in flight deficits between $d F z 2-I R$ and the three other RNAi strains is very likely due to a difference in their efficacy of knockdown (compare Figure 1E with Figure 1-figure supplement 2B). Therefore, the $d F z 2-I R$ strain was used for all subsequent analyses.

\section{$\mathrm{dFz2}$ is required in the PAM dopaminergic neurons for flight}

Dopaminergic neurons marked by THGAL4 have been broadly classified into seven clusters in the brain (Figure 2A; Table 1). In addition TH-expressing neurons are present in each segment of the ventral ganglion (Mao and Davis, 2009; Sadaf et al., 2015). In the brain, two neuronal clusters referred to as PAM and PAL (Protocerebral Anterior Lateral) are located in the anterior region, whereas five neuronal clusters, PPM1, 2, and 3 (Protocerebral Posterior Medial), PPL1 and 2 (Protocerebral Posterior Lateral) are located in the posterior region (Figure 2A). In order to identify 


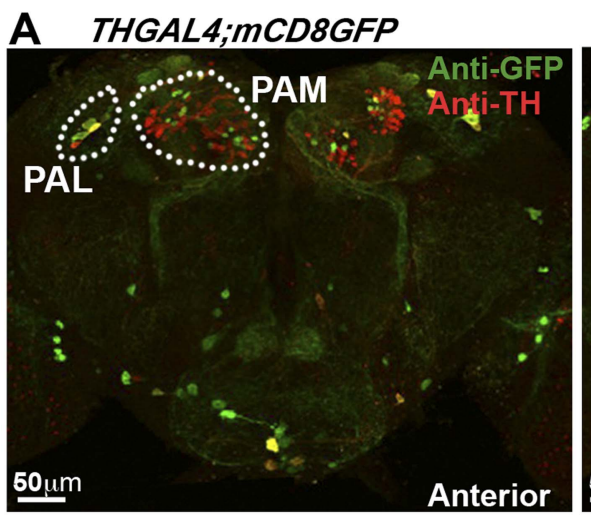

B

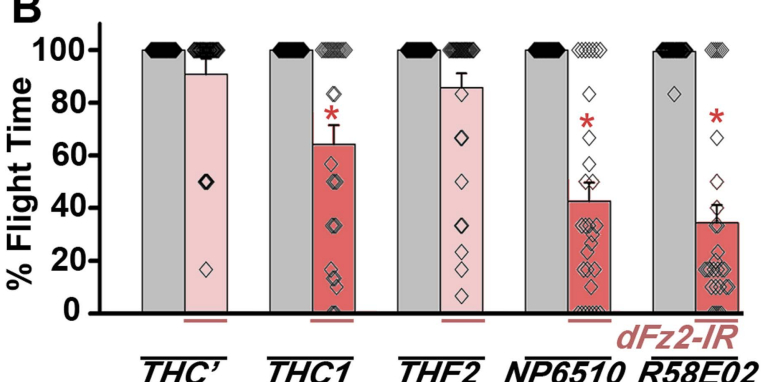

D
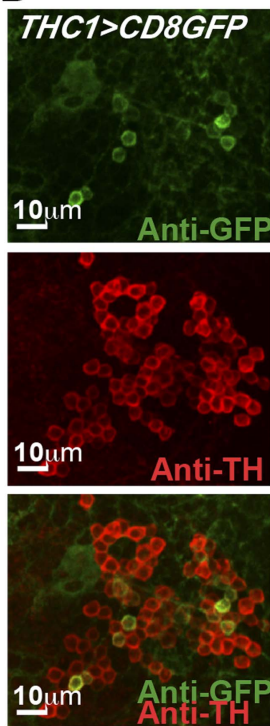
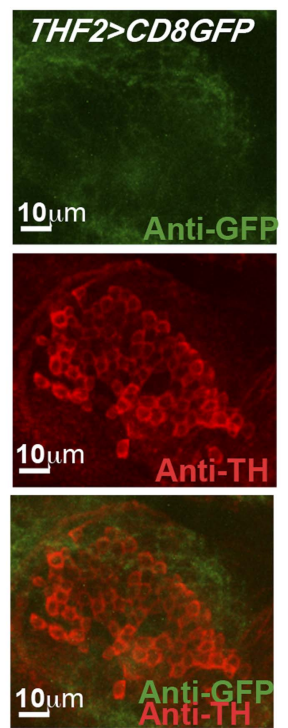

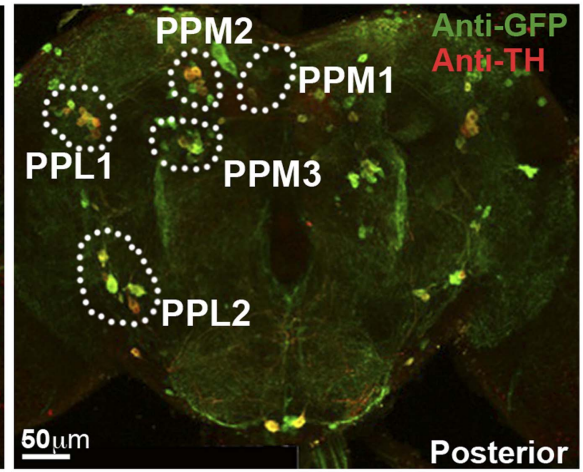

C

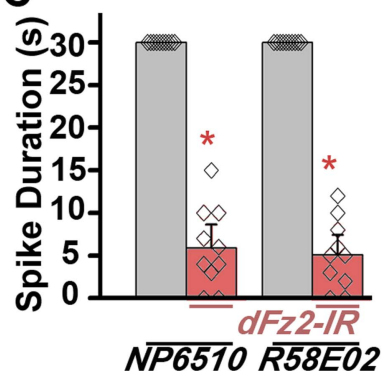

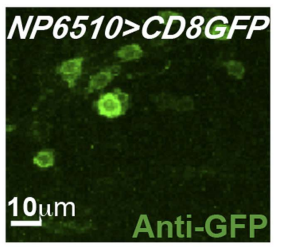
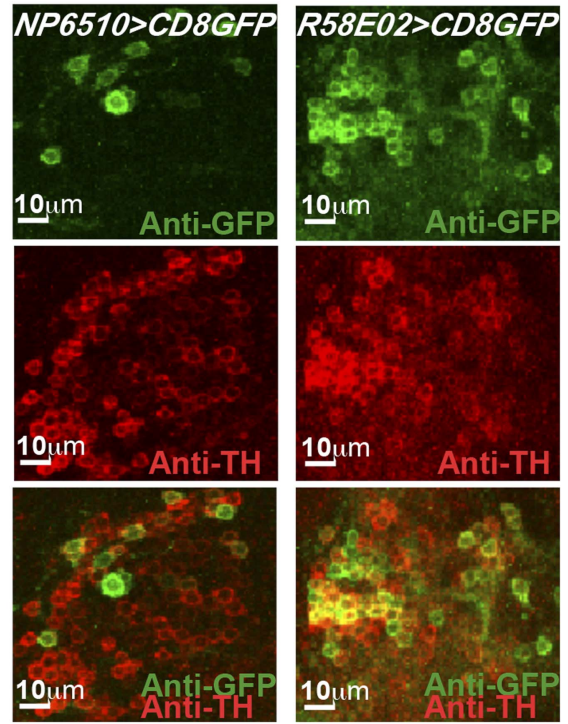

Figure 2. RNAi-mediated knockdown of $d F z 2$ function in Protocerebral Anterior Medial (PAM) dopaminergic neurons causes flight deficits. (A) Expression pattern of THGAL4 in the anterior and posterior regions of the brain are shown. Dotted line markings show the neuronal clusters. PAM: protocerebral anterior medial; PAL: protocerebral anterior lateral; PPM1, PPM2, PPM3: protocerebral posterior medial 1, 2, and 3; PPL1, PPL2: protocerebral posterior lateral 1 and 2. (B) Percentage flight times of heterozygous GAL4 controls (gray bars) and GAL4-specific knockdown of $d F z 2$ (red bars). Knockdown of $d F z 2$ in PAM-expressing GAL4 individuals (THC1GAL4, NP6510GAL4, R58E02GAL4) resulted in significantly reduced flight times when compared to their respective GAL4 controls ( ${ }^{*} p<0.001$, Mann-Whitney U-test). (C) Durations of rhythmic action potentials recorded from the DLMs of air-puff stimulated tethered flies. Average Spike durations were reduced upon expression of $d F z 2$ RNAi in NP6510GAL4 and R58E02GAL4 as compared to GAL4s controls ( ${ }^{*} p<0.001$, Mann-Whitney U-test). (D) Expression of THC1GAL4, THF2GAL4, NP6510GAL4, and R58E02GAL4 in the PAM neuronal cluster is shown. Except Figure 2. continued on next page 
Figure 2. Continued

THF2GAL4, all other GAL4s express in dopaminergic PAM neurons. Expression was analyzed from 10 brain hemispheres.

DOI: 10.7554/eLife.07046.007

The following figure supplements are available for figure 2 :

Figure supplement 1. Electrophysiological traces showed reduced firing upon knockdown of $d F z 2$. DOI: 10.7554/eLife.07046.008

Figure supplement 2. Expression of $\mathrm{dFz} 2$ in PAM dopaminergic neurons. DOI: 10.7554/eLife.07046.009

Figure supplement 3. Expression of TH in PAM dopaminergic neurons during development. DOI: 10.7554/eLife.07046.010

TH-expressing neurons that require dFz2 function for flight, three independent GAL4 strains (THC', THC1, and THF2; Liu et al., 2012), with differential expression in central brain clusters and the ventral ganglion, were tested (Table 1). Significant flight deficits were observed upon expression of $d F z 2-I R$ under control of THC1GAL4, but not with THC'GAL4 and THF2GAL4 (Figure 2B). These data suggested that either all or some neurons in the PAM, PPM1, and T3 regions, marked by THC1GAL4, but poorly marked or not marked by THC'GAL4 and THF2GAL4, form part of the flight circuit and require dFz2 signaling during pupal development. Next, we tested two strains (NP6510GAL4 and R58E02GAL4) (Riemensperger et alo, 2013) which drive expression uniquely in the PAM neurons (Table 1). Significant flight deficits were observed in flies with knockdown of $d F z 2$ by either NP6510GAL4 or R58E02GAL4 (Figure 2B,C, Figure 2-figure supplement 1, Video 2) implicating these dopaminergic neurons as part of a central brain flight circuit. These data do not rule out a role for additional central neurons or ventral ganglion neurons in the regulation of flight. Expression of $\mathrm{dFz} 2$ was confirmed in adult PAM neurons by immunohistochemistry (Figure 2-figure supplement 2). Knockdown by $d F z 2-I R$ in PAM neurons resulted in significant

Table 1. Summary of expression pattern of GAL4s THC' THC1 THF2 NP6510 R58E02

\begin{tabular}{lccccc}
\hline $\mathrm{PAM}$ & + & ++ & - & ++ & +++ \\
\hline $\mathrm{PAL}$ & + & + & - & - & - \\
\hline $\mathrm{PPM} 1$ & + & ++ & - & - & - \\
\hline $\mathrm{PPM} 2$ & + & + & + & - & - \\
\hline $\mathrm{PPM} 3$ & - & - & + & - & - \\
\hline $\mathrm{PPL} 1$ & - & + & ++ & - & - \\
\hline $\mathrm{PPL} 2$ & + & + & + & - & - \\
\hline $\mathrm{T} 1$ & + & + & - & - & - \\
\hline $\mathrm{T} 2$ & - & + & + & - & - \\
\hline $\mathrm{T} 3$ & - & + & - & - & - \\
\hline $\mathrm{Ab}$ & + & + & + & - & -
\end{tabular}

Table summarizing the expression pattern of THC'GAL4, THC1GAL4, THF2GAL4, NP6510GAL4, and R58E02GAL4 in specified dopaminergic neuronal clusters. Clusters shown in Figure $\mathbf{2 A}$ and thoracic ganglion $(T 1, T 2, T 3, A b)$ were examined for the expression. Plus $(+)$ and minus $(-)$ indicate the presence and absence of dopaminergic positive neurons, respectively. Double plus $(++)$ and triple plus $(+++)$ indicate the presence of $>5$ and $>50$ dopaminergic positive neurons, respectively. 10 brain hemispheres were analyzed for the expression

DOI: 10.7554/eLife.07046.011 loss of $\mathrm{dFz} 2$ immunostaining (Figure 2-figure supplement 2). Moreover, in support of the pupal requirement for $\mathrm{dFz} 2$ (Agrawal et al。, 2013) (Figure 1B), PAM neurons marked by R58E02GAL4 do not express $\mathrm{TH}$ in the larval stages (Figure 2-figure supplement 3), indicating that $\mathrm{TH}$ immunoreactivity in these neurons is acquired during pupal maturation, as observed by co-localization of TH immunostaining with R58E02GAL4-driven GFP in pupae (Figure 2-figure supplement 3). With this we concluded that PAM neurons require $\mathrm{dFz} 2$ signaling during functional maturation of the flight circuit in pupae.

\section{dFz2 regulates flight circuit development through non- canonical $\mathbf{F z 2} / \mathrm{Ca}^{2+}$ signaling}

A role for $\mathrm{dFz} 2$ in flight circuit development was originally identified in a screen for GPCRs that signal through changes in intracellular $\mathrm{Ca}^{2+}$ (Agrawal et alo, 2013). In order to identify molecules that function downstream of Fz2 for development of the adult flight circuit, interactions of candidate genes were tested. Reports from vertebrates suggest that $\mathrm{dFz} 2$ activates downstream $\mathrm{Ca}^{2+}$ signaling through non-canonical 


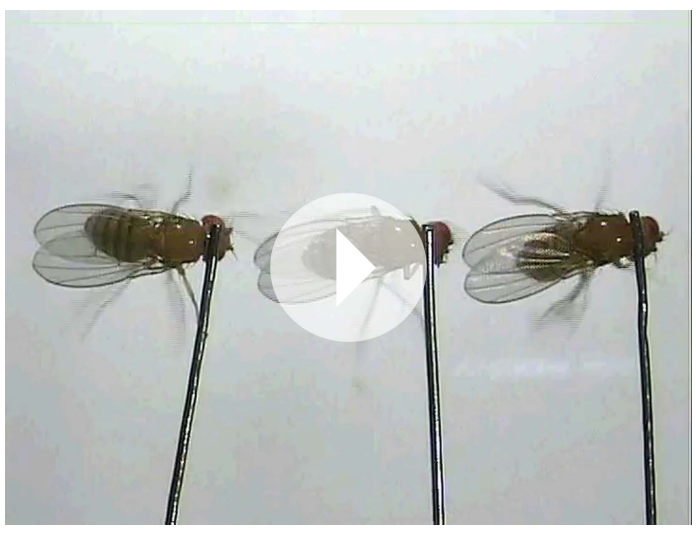

Video 2. dFz2 knockdown in PAM neurons result in flight defect. Real time video recording of air-puff induced flight in the following genotypes from left to right. (1) R58E02GAL4/+, (2) R58E02GAL4; dFz2-IR, (3) $d F z 2-I R /+$. Following a gentle air-puff R58E02GAL4; $d F z 2-I R$ flies were able to initiate but not maintain flight for as long as control flies of the genotypes THGAL4/+ and $d F z 2-I R /+$

DOI: $10.7554 /$ LLife. 07046.012

knockdown of canonical dFz2 pathway components like LRP5/6, Dishevelled, and GSK3 $\beta$ in either the panneuronal domain or in dopaminergic neurons (Figure 3-figure supplement 1). RNAi strains for dishevelled and GSK3 $\beta$ were validated by quantitative PCR (qPCR; Figure 3-figure supplement 1), whereas RNAi for LRP5/6 was validated by ubiquitous expression with Act5CGAL4 that resulted in embryonic lethality (Dietzl et al., 2007). These results do not support a role for canonical dFz2 signaling in dopaminergic neurons for maturation of the flight circuit in pupae.

Next, we tested flight deficits by expression of previously implicated non-canonical candidates that link dFz2 activation with $\mathrm{Ca}^{2+}$ signaling (Figure 3A) (Sheldahl et al., 2003). From genetic studies, we know that the heterotrimeric G-protein, Gq, which links GPCR activation to intracellular store calcium release, does not function downstream of dFz2 signaling in the context of Drosophila flight (Agrawal et al., 2013). Therefore, we tested the requirement of other heterotrimeric G-proteins from Drosophila. Constitutively, active forms of the $\alpha$ subunits of Gs (UASAcGs), Go (UASAcGo), and Gi (UASAcGi) were tested in flies with $d F z 2$ knockdown in pupal stages. Pan-neuronal expression of AcGo in pupae with $d F z 2$ knockdown, rescued flight defects to a significant extent (Figure 3B), whereas constitutively active forms of $\mathrm{Gi}, \mathrm{Gq}$, or $\mathrm{Gs}$ did not (Figure 3-figure supplement 2). A partial rescue of flight defects was also observed upon AcGo expression in dopaminergic neurons in pupae (Figure 3B). These data support Go activation by $\mathrm{dFz} 2$ in dopaminergic neurons of the maturing flight circuit during pupal development.

To confirm the requirement of Go in dopaminergic neurons, we down-regulated Go function either by expression of an RNAi construct (Vecsey et al., 2014) THGAL4;GoRNAi or by expression of pertussis toxin which inhibits Go function (THGAL4;UASPTX.16) and tested the progeny for flight. In Drosophila, pertussis toxin is a selective inhibitor of Go signaling (Hopkins et al., 1988; Ferris et al., 2006). Expression of Go-IR and PTX.16 in dopaminergic neurons reduced both flight times (Figure 3B), and the maintenance time of flight patterns recorded from the DLMs (Figure 3D,E). Moreover, down-regulation of Go signaling in PAM neurons with R58E02GAL4, by expression of either Go-IR or PTX.16 resulted in significant loss of flight (Figure 3B). PTX induced flight deficits required expression in pupae and not in adults (Figure $3 B$ and Figure 3-figure supplement 2).

Flight deficits induced by pan-neuronal knockdown of $d F z 2$ were rescued to a significant extent by over-expression of the ER $\mathrm{Ca}^{2+}$ depletion sensor, dSTIM+ (Agrawal et al., 2013), suggesting that activation of $\mathrm{Go}$ by dFz2 evokes $\mathrm{Ca}^{2+}$ signals in Drosophila neurons. As in other organisms (Feske et al., 2006; Prakriya et al., 2006; Vig et al., 2006), in Drosophila neurons as well $\mathrm{Ca}^{2+}$ release 


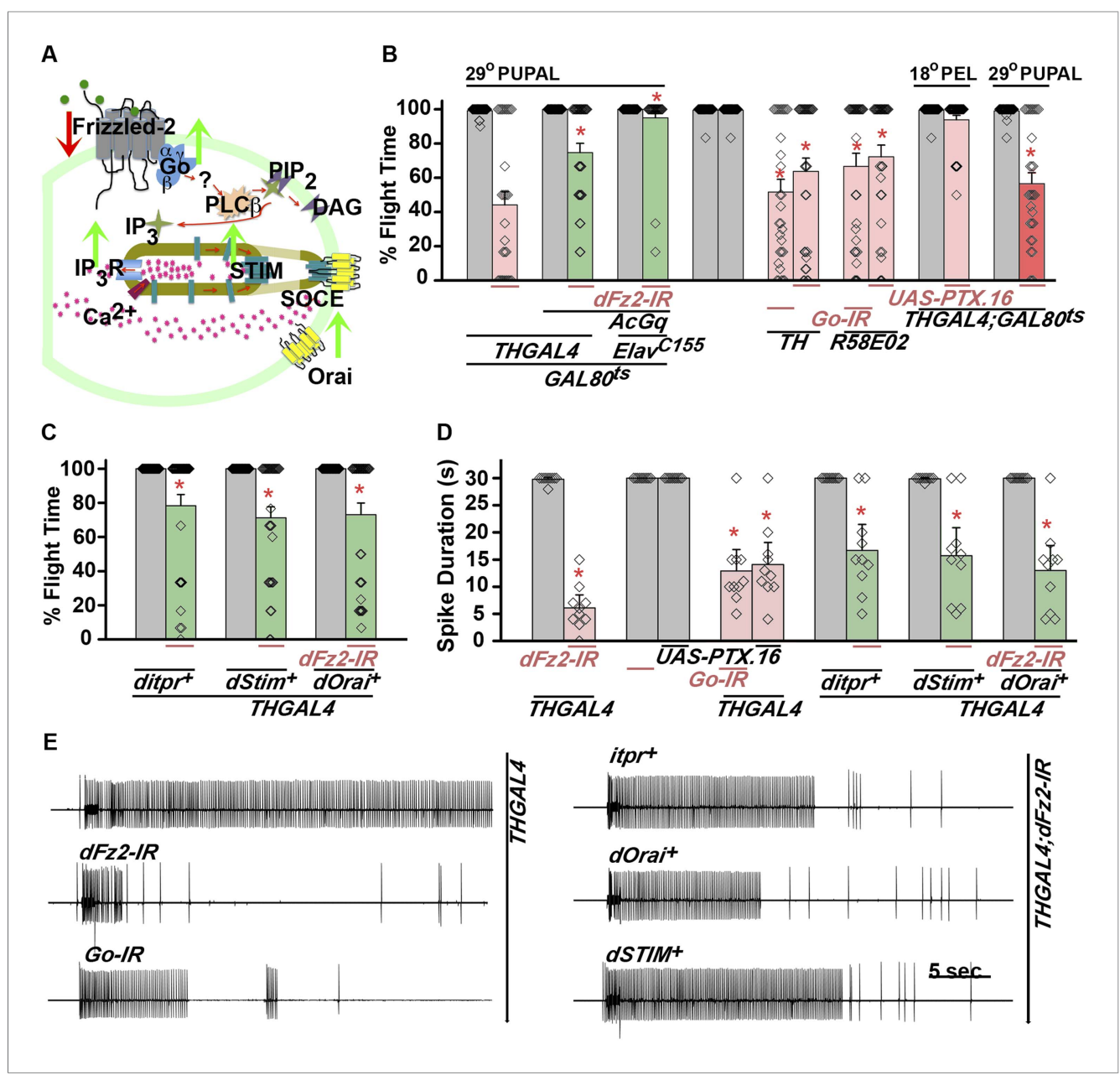

Figure 3. $d F z 2$ function is mediated through $\mathrm{G}$-protein $\mathrm{Go}$ and $\mathrm{IP}_{3}$-mediated calcium signaling in dopaminergic neurons. (A) A schematic showing dFz2-mediated activation of Go followed by $\mathrm{P}_{3} \mathrm{R}$-mediated $\mathrm{Ca}^{2+}$ signaling pathway and Store-operated $\mathrm{Ca}^{2+}$ entry (SOCE) through dSTIM and dOrai. Red (down-regulation) and green (overexpression) arrows indicate the two strategies used for testing this signaling mechanism. (B) Percentage flight times of the indicated genotypes are shown. Knockdowns flight times were compared to their respective heterozygote controls, whereas AcGo rescue of dFz2 knockdown was compared to dFz2 knockdown ( ${ }^{*} p<0.001$, Mann-Whitney U-test). (C) Percentage flight times of heterozygous controls (gray bars) followed by over-expression of calcium signaling molecules (itpr ${ }^{+}, d \mathrm{Stim}^{+}, \mathrm{dOrai}{ }^{+}$) in flies with knockdown of $d F z 2$ (green bars). Overexpression of calcium signaling molecules (itpr $\left.{ }^{+}, d S t i m^{+}, d O r a i^{+}\right)$rescued flight defects significantly when compared to flies with $d F z 2$ knockdown ( ${ }^{*} p<0.001$, Mann-Whitney U-test). (D) Durations of rhythmic action potentials recorded from the DLMs of air-puff stimulated tethered flies. Spike durations were reduced upon expression of Go RNAi or UAS-PTX.16 in dopaminergic neurons and partially rescued upon over-expression of calcium signaling molecules (itpr ${ }^{+}$, dStim $^{+}$, $d \mathrm{Orai}^{+}$) when compared to knockdown of $d F z 2$ ( ${ }^{*} p<0.001$, Mann-Whitney U-test). (E) Representative electrophysiological recordings from DLMs of the indicated genotypes.

DOI: 10.7554/eLife.07046.013

The following figure supplements are available for figure 3:

Figure supplement 1. The canonical Fz2/ $\beta$ catenin signaling pathway does not function downstream of $d F z 2$ in the context of flight circuit maturation.

DOI: 10.7554/eLife.07046.014

Figure supplement 2. Go functions downstream of dFz2 in the context of flight circuit maturation. DOI: 10.7554/eLife.07046.015

Figure supplement 3. Non-canonical $\mathrm{dFz} / \mathrm{Ca}^{2+}$ signaling functions downstream of $\mathrm{dFz} 2$ in the context of flight circuit maturation.

DOI: 10.7554/eLife.07046.016 
through the $\mathrm{IP}_{3} \mathrm{R}$ leads to clustering of $\mathrm{dSTIM}$, which in turn promotes Store-operated $\mathrm{Ca}^{2+}$ entry (SOCE) through dOrai (Venkiteswaran and Hasan, 2009; Agrawal et al., 2010) (Figure 3A). In a converse experiment, we tested the effect of over-expression of a $\mathrm{dFz} 2^{+}$transgene on flight deficits induced by pan-neuronal knockdown of the $I_{3} R$ (itpr-IR), dSTIM (dSTIM-IR) and dOrai (dOrai-IR) and observed a significant rescue in all three conditions tested (Figure 3-figure supplement 3, Video 3, and Video 4). These data suggest that, as in vertebrate neurons, dFz2 links to intracellular calcium signaling in Drosophila. Next, we tested the effect of over-expression of $\mathrm{dSTIM}^{+}$, on flight deficits induced by $d F z 2$ knockdown in dopaminergic neurons. A partial but significant rescue of flight was observed (Figure $3 \mathrm{C}$ ) accompanied by a rescue of the duration of firing patterns from the DLMs (Figure 3D,E). Over-expression of the $\mathrm{IP}_{3} \mathrm{R}$ and dOrai also rescued flight deficits observed by knockdown of $\mathrm{dFz} 2$ in dopaminergic neurons (Figure 3C,D,E). Together these data support the idea that maturation of dopaminergic neurons in the flight circuit requires intracellular $\mathrm{Ca}^{2+}$ signaling by activation of $\mathrm{dFz} 2$ and Go. The mechanism by which Go activates $\mathrm{Ca}^{2+}$ signaling through $\mathrm{IP}_{3} \mathrm{R} / \mathrm{dSTIM}$ requires further investigation (Figure $3 \mathrm{~A}$ ).

\section{Down-regulation of dFz2 affects neuronal activity in maturing PAM neurons required for adult flight}

Synaptic function of developing hippocampal neurons can be modulated by $\mathrm{Ca}^{2+}$ signaling downstream of Fz2 (Varela-Nallar et al., 2010). In Drosophila, neuronal activity can be increased by over-expression of a voltage-gated sodium channel, $\mathrm{NaChBac}$ (Nitabach et al., 2006). Therefore, we tested flight in organisms with $d F z 2$ knockdown and increased neuronal activity by $\mathrm{NaChBac}$ expression. Flight was restored close to $100 \%$ upon expression of $\mathrm{NaChBac}$ in dopaminergic neurons (THGAL4) and more specifically in PAM neurons (NP6510GAL4 and R58E02GAL4; Figure 4A, Video 5). Moreover, raising neuronal activity during pupal development in parallel with $d F z 2$ knockdown compensated for loss of flight observed in the knockdown condition (Figure 4B). These data suggest that $\mathrm{Fz} 2 / \mathrm{Ca}^{2+}$ signaling can contribute to the synaptic activity of dopaminergic PAM neurons in pupae. The requirement for synaptic activity in maturing PAM neurons was tested directly by expression of a temperature sensitive mutant of the dynamin orthologue, shibire ${ }^{t s}\left(S_{i} i^{t s}\right)$. Expression of Shits blocks vesicle endocytosis at $30^{\circ} \mathrm{C}$ (Kitamoto, 2001) and its expression during pupal development, either in $\mathrm{TH}$ neurons (THGAL4) or exclusively in PAM neurons (NP6510GAL4 and R58E02GAL4) resulted in significant loss of flight (Figure 4C). Temporal expression of Shits in adult PAM neurons also resulted in a flight deficit (Figure 4D), supporting the requirement of active synaptic transmission in PAM neurons for adult flight.

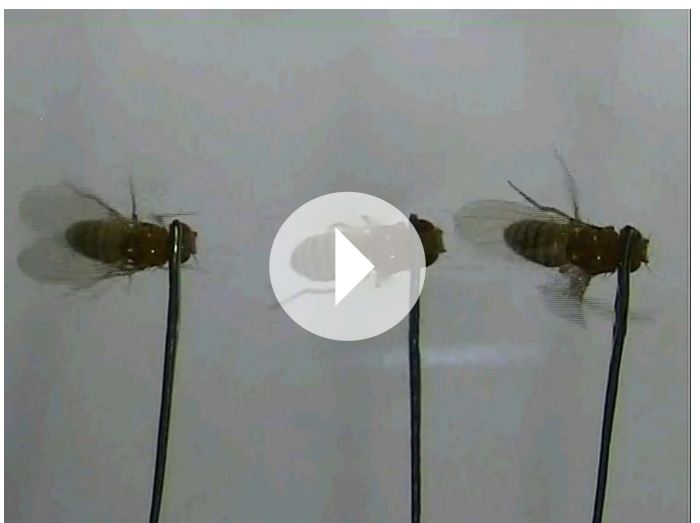

Video 3. Overexpression of $\mathbb{P}_{3} R$ in dopaminergic neurons rescues flight defects of $\mathrm{dFz} 2$ downregulation. Real time video recording of air-puff induced flight in the following genotypes from left to right. (1) THGAL4; $d F z 2-I R$;itpr ${ }^{+}$, (2) THGAL4;dFz2-IR, (3) dFz2-IR/+.

Following a gentle air-puff THGAL4; $d F z 2-I R ;$ itpr $r^{+}$flies were able to initiate and maintain flight for a longer duration as compared to THGAL4;dFz2-IR.

DOI: 10.7554/eLife.07046.017

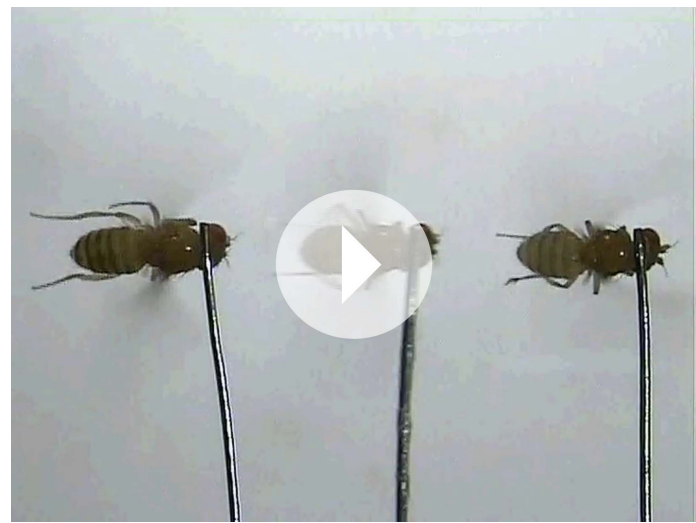

Video 4. Flight defects in dFz2 knockdown individuals can be rescued by over-expression of dSTIM. Real time video recording of air-puff induced flight in the following genotypes from left to right. 1) THGAL4; $d F z 2-I R$; $\left.d S T I M^{+}, 2\right)$ THGAL4; $d F z 2-I R, 3) d F z 2-I R /+$. Following a gentle air-puff THGAL4; dFz2-IR; dSTIM+ flies were able to initiate and maintain flight for a longer duration as compared to THGAL4; dFz2-IR.

DOI: $10.7554 /$ eLife.07046.018 


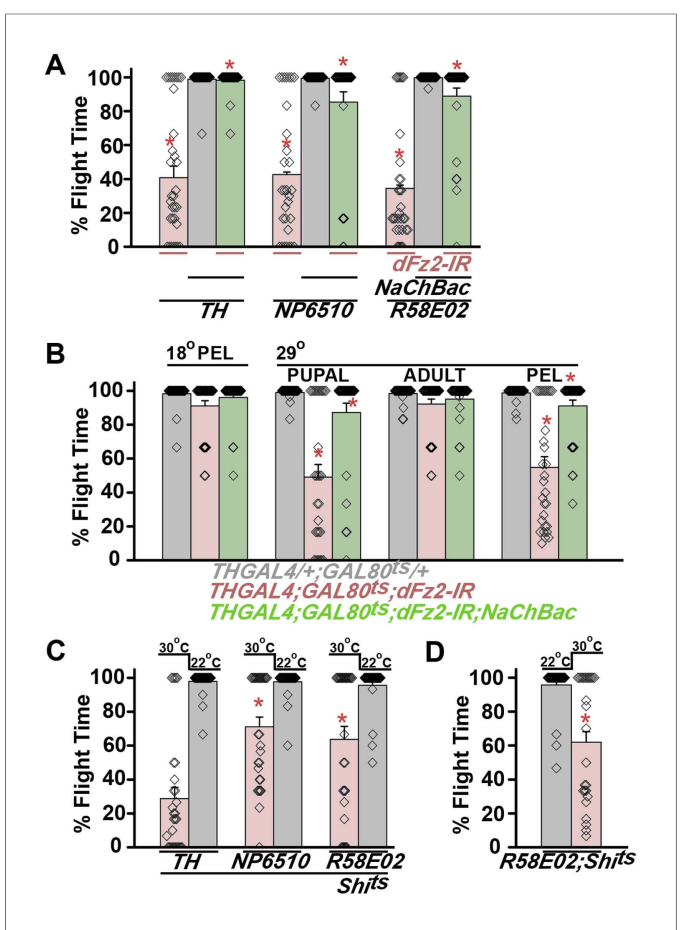

Figure 4. Knockdown of $\mathrm{dFz} 2$ affects neuronal activity of maturing flight circuit PAM neurons. (A) Percentage flight times of individual heterozygous controls (gray bars), dFz2 knockdown ( $d F z 2-I R$ ) in dopaminergic neurons (THGAL4) and PAM neurons (NP6510GAL4, R58E02GAL4) (red bars) followed by over-expression of $\mathrm{NaChBac}$ in presence of $d F z 2-I R$ (green bars); ( ${ }^{*} p<0.001$, Mann-Whitney U-test). (B) Percentage flight times for heterozygotes of THGAL4;GAL80 (gray bars) followed by stage-specific knockdown of $d F z 2$ (red bars) and over-expression of $\mathrm{NaChBac}$ in flies with $d F z 2$ knockdown (green bars) as indicated. Over-expression of $\mathrm{NaChBac}$ during pupal development rescued flight as did over-expression post-egg laying (PEL) ${ }^{*} p<0.001$, Mann-Whitney U-test). (C) Percentage flight times upon expression of Shibire ${ }^{\text {ts }}\left(\mathrm{Sh}^{\text {ts }} 30^{\circ} \mathrm{C}\right.$; red bars) either in pupal or no expression ( $S h^{\text {ts }} 22^{\circ} \mathrm{C}$; gray bars). Expression was either in dopaminergic neurons (THGAL4) or PAM neurons (NP6510GAL4, R58E02GAL4). Flight was tested at $25^{\circ} \mathrm{C}$. Expression of $\mathrm{Sh}^{\text {ts }}$ in pupal resulted in reduced flight times. (D) Percentage flight times upon adult expression of Shibire ${ }^{\text {ts }}$ (Shit $30^{\circ} \mathrm{C}$; red bars) or no expression ( $\mathrm{Sh}^{\text {ts }} 22^{\circ} \mathrm{C}$; gray bars), in PAM neurons with R58E02GAL4. Flight was tested at $30^{\circ} \mathrm{C}$. Expression of Shits resulted in reduced flight times $\left({ }^{*} p<0.001\right.$, Mann-Whitney U-test).

DOI: 10.7554/eLife.07046.019

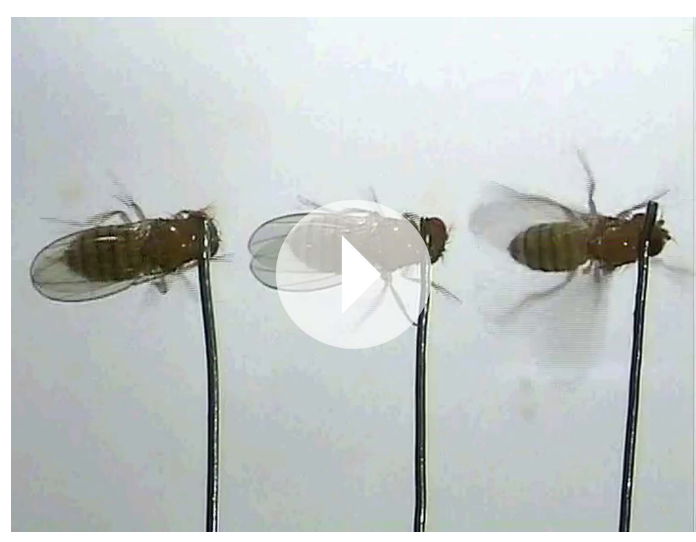

Video 5. Increased neuronal activity in PAM neurons rescues flight in individuals with $\mathrm{dFz} 2$ knockdown. Real time video recording of air-puff induced flight in the following genotypes from left to right. (1) R58E02GAL4; dFz2-IR;NaChBac, (2) R58E02GAL4;dFz2-IR, (3) dFz2-IR/+. Following a gentle air-puff R58E02GAL4; $d F z 2-I R$; $\mathrm{NaChBac}$ flies were able to initiate and maintain flight for a longer duration as compared to R58E02GAL4; dFz2-IR.

DOI: 10.7554/eLife.07046.020

\section{dFz2 is required in PAM neurons for normal expression of TH}

The cellular effect of reduced $d F z 2$ expression in PAM neurons was investigated next. TH levels in PAM neurons marked by R58E02GAL4, appear reduced upon $d F z 2$ knockdown as judged by immunohistochemistry (compare anti-TH panels in Figure $5 \mathrm{~A}, \mathbf{B}$ ). Expression of $\mathrm{NaChBac}$ with $d F z 2-I R$ restored $\mathrm{TH}$ expression close to wildtype levels (Figure $5 \mathrm{~A}, \mathrm{~B}, \mathrm{C}$ ). Quantification of $\mathrm{TH}$ immunostaining across multiple samples revealed a significant reduction upon $d F z 2$ knockdown which was restored by expression of $\mathrm{NaChBac}$ (Figure 5D,E). Furthermore, $\mathrm{TH}$ transcript levels were significantly reduced by $d F z 2$ knockdown and were restored upon expression of $\mathrm{NaChBac}$ (Figure 5-figure supplement 1). Thus, altered TH levels corroborated well with flight deficits and their rescue in various genotypes. Numbers of TH-positive neurons in the PAM cluster were not significantly different between the three genotypes as judged by anti-TH immunostaining (Figure 5G). Surprisingly, R58E02GAL4-driven GFP expression was also reduced upon expression of $d F z 2-I R$ and was restored back upon expression of $\mathrm{NaChBac}$ (Figure 5-figure supplement 1). Consequently, there was an apparent reduction in the numbers of GFP positive cells upon $d F z 2-I R$ expression which was restored by $\mathrm{NaChBac}$ expression (Figure 5F). Because the number of $\mathrm{TH}$-expressing cells of the PAM cluster remained unchanged upon $d F z 2$ knockdown and after $\mathrm{NaChBac}$ rescue (Figure $5 \mathrm{G}$ ), we hypothesized that $\mathrm{dFz} 2 / \mathrm{Ca}^{2+}$ signaling regulates $T H$ expression in PAM neurons during pupal development. 


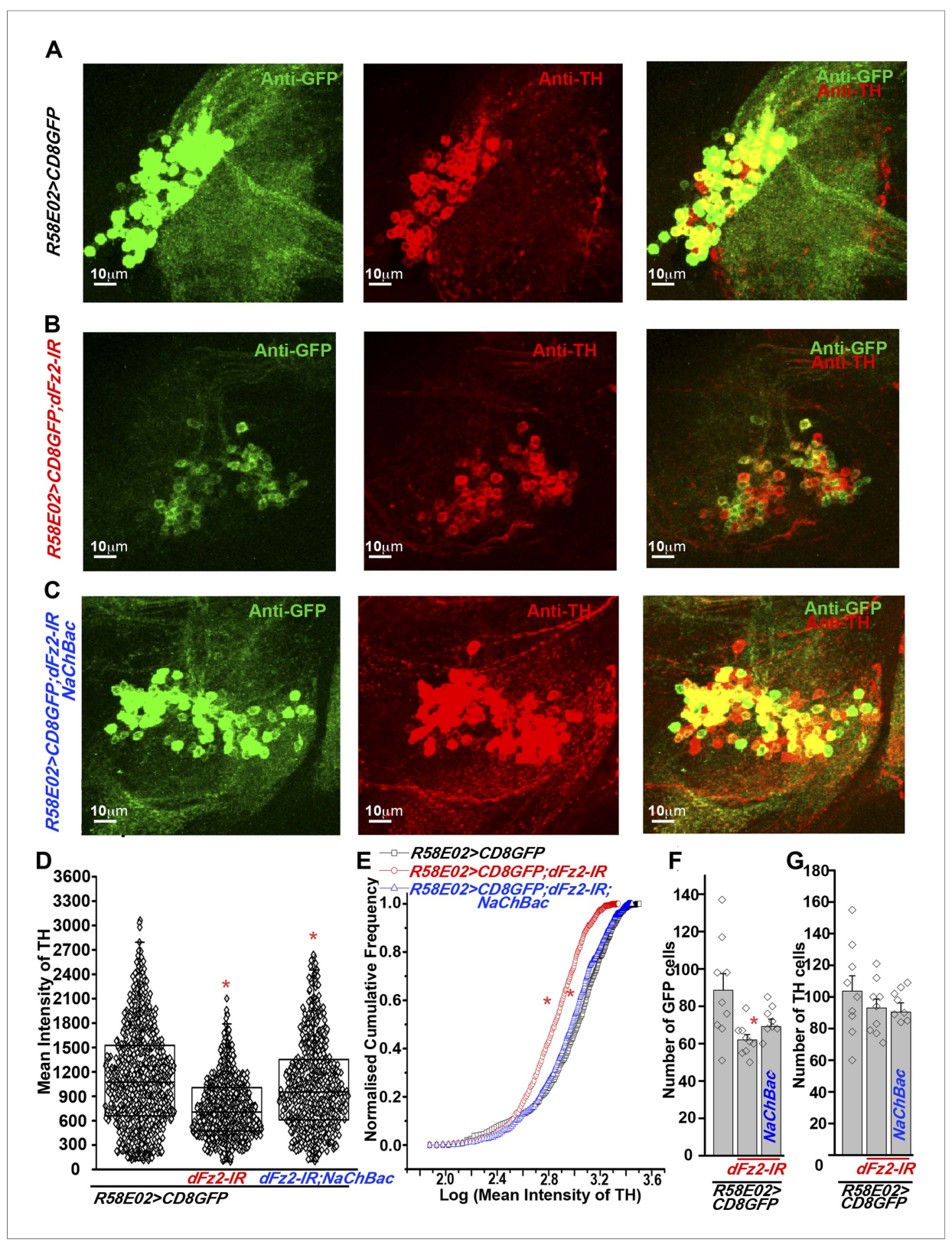

Figure 5. Expression of TH is reduced in PAM neurons by dFz2 knockdown. (A) Expression of GFP (Anti GFP; green) and TH (Anti TH; red) is shown in PAM dopaminergic neurons marked by R58E02GAL4>mCD8GFP. (B) Significant reduction of GFP and TH immunoreactivity is observed in PAM neurons of R58E02GAL4>mCD8GFP; dFz2-IR individuals; which is (C) rescued by over-expression of NaChbac (R58E02GAL4> mCD8GFP; dFz2-IR;NaChBac). (D) Scatter plot with the mean intensity of TH expression in individual PAM neurons $(N=1280)$ in the indicated genotypes. Cells were obtained from 16 brain hemispheres; ${ }^{\star} p<0.05$, one-way ANOVA. (E) A Kolmogorov-Smirnov (K-S) plot analyzing the distribution of the mean intensity of TH immunoreactivity in PAM neurons. The frequency distribution is significantly shifted to the left for R58E02GAL4>mCD8GFP;dFz2-IR as compared to

R58E02GAL4>mCD8GFP indicating a significantly higher percentage of cells with lower mean intensity. Frequency distribution of R58E02GAL4> mCD8GFP; $d F z 2-I R ; N a C h B a c$ is shifted back towards the control distribution R58E02GAL4>mCD8GFP, indicating a significant rescue $\left({ }^{*} \mathrm{P}_{\mathrm{K}-\mathrm{S}}<0.05\right)$. (F) Total number of GFP positive cells and (G) TH positive cells were counted in the indicated genotypes. No difference in the number of TH cells was found; however GFP cells were reduced upon dFz2 knockdown ( ${ }^{\star} p<0.05$, one-way ANOVA).

DOI: 10.7554/eLife.07046.021

The following figure supplements are available for figure 5 :

Figure supplement 1. Expression of GFP is altered upon expression of $d F z 2-I R$ in PAM neurons using R58E02GAL4. DOI: 10.7554/eLife.07046.022

Figure 5. continued on next page 
Figure 5. Continued

Figure supplement 2. Altered GFP expression was seen upon expression of $d F z 2-I R$ in dopaminergic neurons. DOI: 10.7554/eLife.07046.023

Figure supplement 3. Knockdown of dFz2 in OK371GAL4-expressing neurons does not affect TH expressionpositive PAM neurons.

DOI: 10.7554/eLife.07046.024

Moreover, our data support a role for $\mathrm{dFz} 2 / \mathrm{Ca}^{2+}$ signaling in regulating expression of the R58E02GAL4 transgene where GAL4 is under control of the fumin gene encoding a Dopamine Transporter, DAT (Liu et al., 2012).

The status of TH and GFP expression in PAM neurons was further investigated after $d F z 2$ knockdown with THGAL4. Unlike R58E02GAL4, THGAL4-driven mGFP marks a small subset of PAM neurons. This consists of two clusters of 6-7 neurons each (Figure 2A and Figure 5-figure supplement 2) (Riemensperger et al., 2013). THGAL4-driven expression of dFz2-IR resulted in significant loss of GFP expression in PAM neurons (Figure 5-figure supplement 2). Interestingly, GFP expression in five TH-positive neurons of the PAL cluster remained unaffected by knockdown of $d F z 2$, suggesting that $d F z 2$ regulation of $T H$ expression maybe PAM specific (data not shown). Overexpression of either $d S T I M^{+}, I P_{3} R$ or $N a C h B a c$ in the background of $d F z 2$ knockdown could partially rescue loss of GFP expression in the PAM neurons (Figure 5-figure supplement 2). Thus, THGAL4driven GFP expression in PAM neurons correlated with flight deficits and their rescue (Figures 1A, 3C, 4A). Quantification of TH immunoreactivity in PAM cells by THGAL4-driven dFz2 knockdown was technically not possible because the few THGAL4-positive cells of the PAM cluster could not be identified in the $d F z 2$ knockdown condition. Taken together these data support the idea that dFz2/ $\mathrm{Ca}^{2+}$ signaling in PAM neurons drives transcription of two key dopamine synthesis and uptake molecules, TH and DAT. The transcriptional regulation extends to GAL4 transgenic constructs containing TH and DAT regulatory sequences. As controls we tested TH immunoreactivity of PAM neurons in flies with $\mathrm{dFz} 2$ knockdown in glutamatergic neurons (OK371GAL4). Both TH immunoreactivity of PAM neurons (Figure 5-figure supplement 3) and flight patterns (Figure 1-figure supplement 1) were similar to controls.

\section{Altered levels of TH in the dopaminergic PAM neurons cause flight deficits} Based on our observation that $d F z 2$ knockdown in PAM neurons leads to flight deficits accompanied by a significant reduction of $\mathrm{TH}$ expression, we tested the requirement of TH in PAM neurons for flight. Over-expression of a neuronal-specific TH cDNA transgene (UASDTH1) (Friggi-Grelin et al., 2003) in flies with dFz2 knockdown by PAMGAL4 strains (NP6510GAL4 and R58E02GAL4) could rescue flight deficits significantly (Figure 6A, Video 6, Figure 6-figure supplement 1). Furthermore, knockdown of TH with an RNAi (dTH-IR) resulted in significant loss of flight and reduced TH expression (Figure 6A-C,F, Figure 6-figure supplement 1). Moreover, knockdown of TH in PAM neurons affected R58E02GAL4-driven GFP expression suggesting feedback regulation of dopamine transporter (DAT) by dopamine levels. Over-expression of the DTH1 neuronal cDNA could rescue TH immunoreactivity in the R58E02GAL4-expressing PAM neurons with dFz2 knockdown (Figure 6B,C, E). However, GFP immunoreactivity remained low and unchanged between $d F z 2-I R-$ and $d F z 2-I R$; DTH1-expressing PAM neurons (Figure 6-figure supplement 1). These data suggest that rescue of flight by over-expression of DTH1 by passes the transcriptional regulation of DAT by dopamine and of endogenous $\mathrm{TH}$ by $\mathrm{dFz} 2 / \mathrm{Ca}^{2+}$ signaling. They confirm the requirement for $\mathrm{TH}$ expression in PAM neurons for flight.

\section{Maintenance of acute flight requires synaptic activity in $\alpha^{\prime} \beta^{\prime}$ lobes of the mushroom body}

PAM neurons send projections to the horizontal lobes of the MB neuropil (Aso et al., 2012; Burke et al., 2012; Liu et al., 2012; Riemensperger et al., 2013). The MB is a paired brain structure that controls several higher brain functions in insects ranging from olfactory memory formation and reinforcement (Kahsai and Zars, 2011; Waddell, 2013) to locomotor activity (Helfrich-Forster et al., 2002; Serway et al., 2009; Riemensperger et alı, 2013). Axons and dendrites of Kenyon cells, positioned in the calyx region form the MB neuropil which is subdivided into the $\alpha, \beta, \alpha^{\prime}, \beta^{\prime}$, and $\gamma$ lobes 


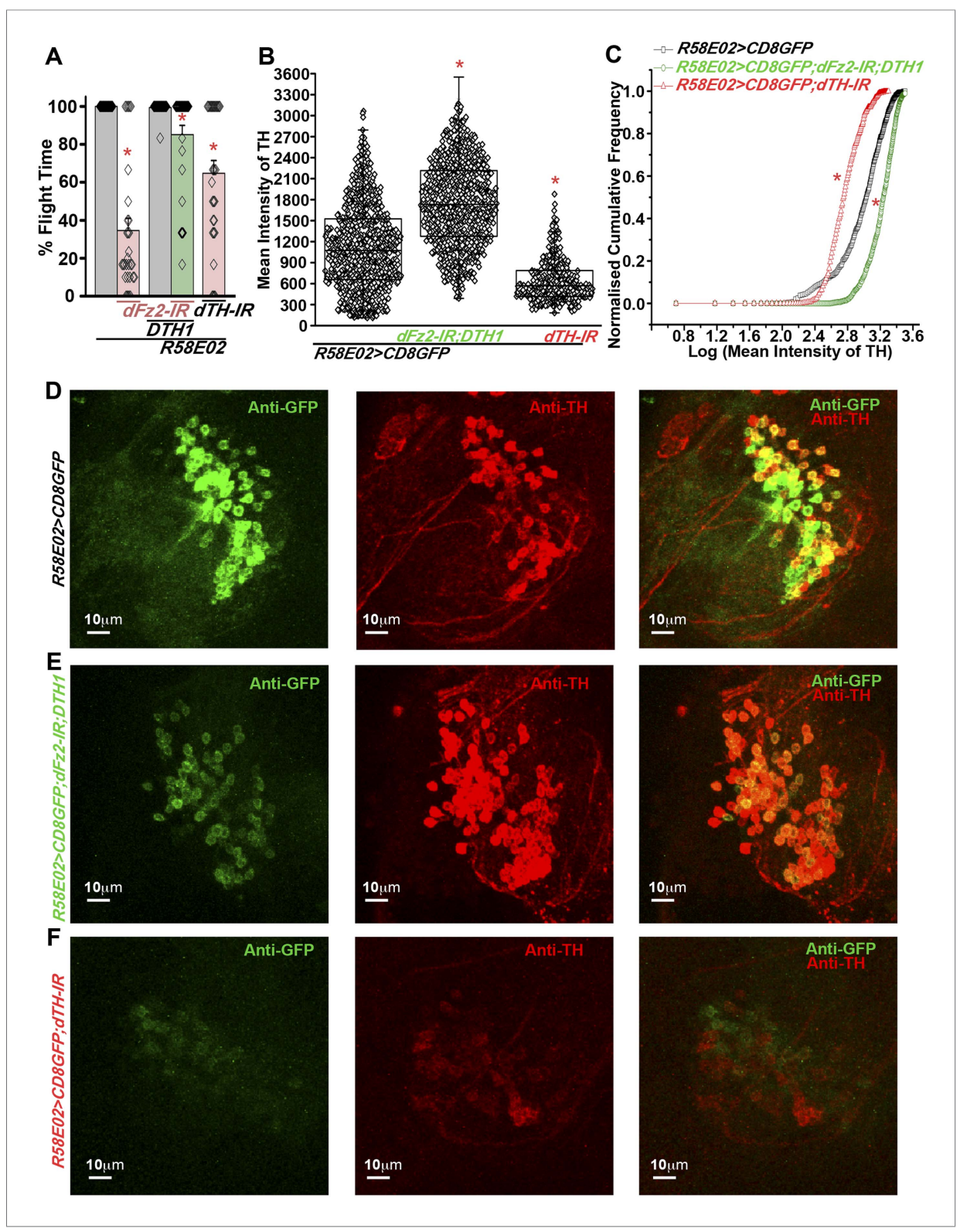

Figure 6. Expression of DTH1 in PAM neurons rescues flight defects shown by dFz2 knockdown. (A) Percentage flight times of individual heterozygous control flies (gray bars), flies with expression of $d F z 2-I R$ and THRNAi (dTH-IR) in PAM neurons (R58E02GAL4) (red bars), and flies with over-expression of DTH1 in the presence of dFz2-IR (green bars). Expression of DTH1 rescued the flight defect of dFz2 knockdown flies to a significant extent $\left({ }^{*} p<0.001\right.$, Mann-Whitney U-test). (B) Scatter plot of the mean intensity of TH expression in individual PAM neurons $(\mathrm{N}=1280)$ from 16 brain hemispheres of the indicated genotypes ( ${ }^{\star} p<0.05$, one-way ANOVA). (C) Kolmogorov-Smirnov (K-S) plot analyzing the distribution of the cellular mean intensity shown in $\mathbf{B}$. The frequency distribution is significantly shifted to the left for R58E02GAL4> mCD8GFP;dTH-IR as compared to R58E02GAL4>mCD8GFP indicating a higher number of cells with lower mean intensity of TH. Frequency distribution of R58E02GAL4> mCD8GFP; dFz2-IR;DTH1 is shifted back to the right indicating fewer cells with lower mean intensity ( ${ }^{*} \mathrm{P}_{\mathrm{K}-\mathrm{S}}<0.05$ ). (D) Expression of GFP (Anti GFP; green) and TH (Anti TH; red) is shown in PAM dopaminergic neurons in R58E02GAL4>mCD8GFP,

(E) R58E02GAL4>mCD8GFP; dFz2-IR; DTH1 and (F) R58E02GAL4> mCD8GFP;dTH-IR. DOI: 10.7554/eLife.07046.025

The following figure supplement is available for figure 6:

Figure supplement 1. Expression of GFP is altered in PAM neurons upon knockdown of $d F z 2$ in the presence of either DTH1 or dsDTH.

DOI: 10.7554/eLife.07046.026 


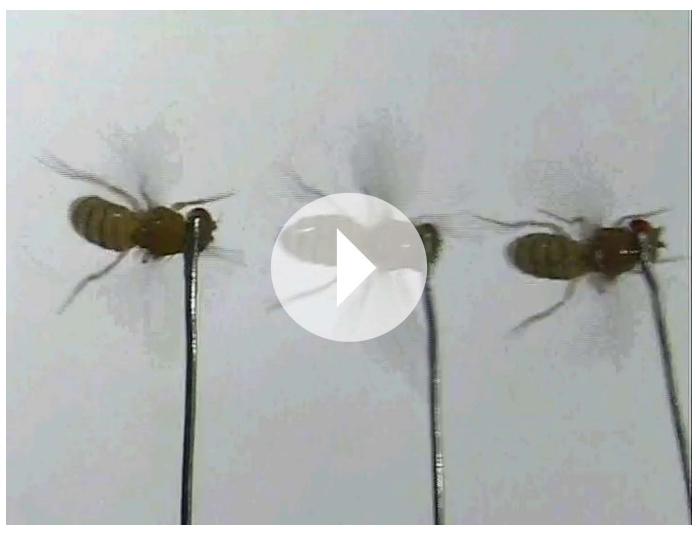

Video 6. DTH over-expression in PAM neurons rescues flight in individuals with $\mathrm{dFz} 2$ knockdown. Real time video recording of air-puff induced flight in the following genotypes from left to right. (1) R58E02GAL4; dFz2-IR;DTH1, (2) R58E02GAL4;dFz2-IR, (3) dFz2-IR/+. Following a gentle air-puff R58E02GAL4; dFz2-IR;DTH1, flies were able to initiate and maintain flight for a longer duration as compared to R58E02GAL4;dFz2-IR. DOI: 10.7554/eLife.07046.027

for post-synaptic dopamine receptors in MB neurons that function for maintenance of acute flight. We tested this requirement further by RNAi-mediated knockdown of the four dopamine receptors-DopECR (CG18314), Dop1R1 (CG9652), Dop1R2 (CG18741), and Dop2R (CG33517), in either the $\alpha^{\prime}, \beta^{\prime}$ neurons (mb186bGAL4) or the $\alpha, \beta, \gamma$ neurons (mb247GAL4; Figure 7-figure supplement 1). A reduction in the length of flight bouts was observed specifically upon knockdown of Dop1R2 in the $\alpha^{\prime}, \beta^{\prime}$ neurons (Figure 7A). The role of $\alpha^{\prime} \beta^{\prime}$ lobes, in flight was supported by another GAL4 driver, c305GAL4, which expresses in the $\alpha^{\prime} \beta^{\prime}$ lobes and faintly in the $\gamma$ lobe (Krashes et al., 2007; Pech et al., 2013). Blocking synaptic activity or knockdown of Dop1R2 using c305aGAL4 resulted in significant flight deficits (Figure 7-figure supplement 2).

Unlike dopaminergic neurons located in the ventral ganglion, which directly modulate flight motor neuron function as demonstrated recently (Sadaf et al., 2015), the PAM-MB circuit described here is not known to project to flight motor neurons in the ventral ganglion (Riemensperger et al., 2013). Rather, PAM-MB circuits function to reinforce both aversive and appetitive olfactory responses (Waddell, 2013). To test possible re-inforcement of flight time by the PAM-MB circuit, we monitored longer flight bouts in several genotypes. Knockdown of $d F z 2$ in PAM neurons (R58E02GAL4>dFz2-IR) significantly reduced the duration of flight bouts, monitored up to $15 \mathrm{~min}$, from an average of $13.04 \pm 0.4$ $\mathrm{min}$ in controls to $0.85 \pm 0.3 \mathrm{~min}$ in the knockdowns (Figure 7C). Maintenance of flight bouts was rescued significantly upon increasing neuronal activity by expression of $\mathrm{NaChBac}$ (Figure $7 \mathrm{C}$ ). These data were analyzed further by binning flight bouts in 20-s intervals (Figure 7D). Flight time of $d F z 2$ knockdown flies clustered towards the left among shorter flight bouts, whereas control flies clustered towards the right with longer flight bouts (Figure 7D). Distribution of flight times in NaChBac-rescued flies appeared intermediate. All rescued flies flew for longer than $20 \mathrm{~s}$ and a small percentage flew for longer than $10 \mathrm{~min}$. Interestingly, DTH1-rescued flies exhibit shorter flight bouts as compared with $\mathrm{NaChBac}$-rescued organisms (Figure $7 \mathrm{C}, \mathrm{D}$ ). This difference in rescue abilities may in part be due to the previous observation that $\mathrm{NaChBac}$ rescue restores $\mathrm{TH}$ immunoreactivity to PAM neurons (Figure 5C), whereas TH rescue very likely cannot restore the excitability deficit of PAM neurons. Long flight bouts were also tested in flies with dFz2 knockdown by THGAL4, followed by rescue with dSTIM ${ }^{+}$, itpr ${ }^{+}$, and $\mathrm{NaChBac}$ (Figure 7-figure supplement 4). The $\mathrm{NaChBac}$ rescue profile was very similar to that observed by $\mathrm{NaChBac}$ rescue of PAM-specific dFz2 knockdown, whereas, dSTIM ${ }^{+}$and itpr ${ }^{+}$ rescue profiles resembled the DTH1 rescue in Figure $7 C_{,} D$ (Figure 7-figure supplement 5). These data support a role for the PAM-MB circuit in maintenance of long flight bouts through dopaminergic synapses on $\alpha^{\prime} \beta^{\prime}$ MB lobes. 


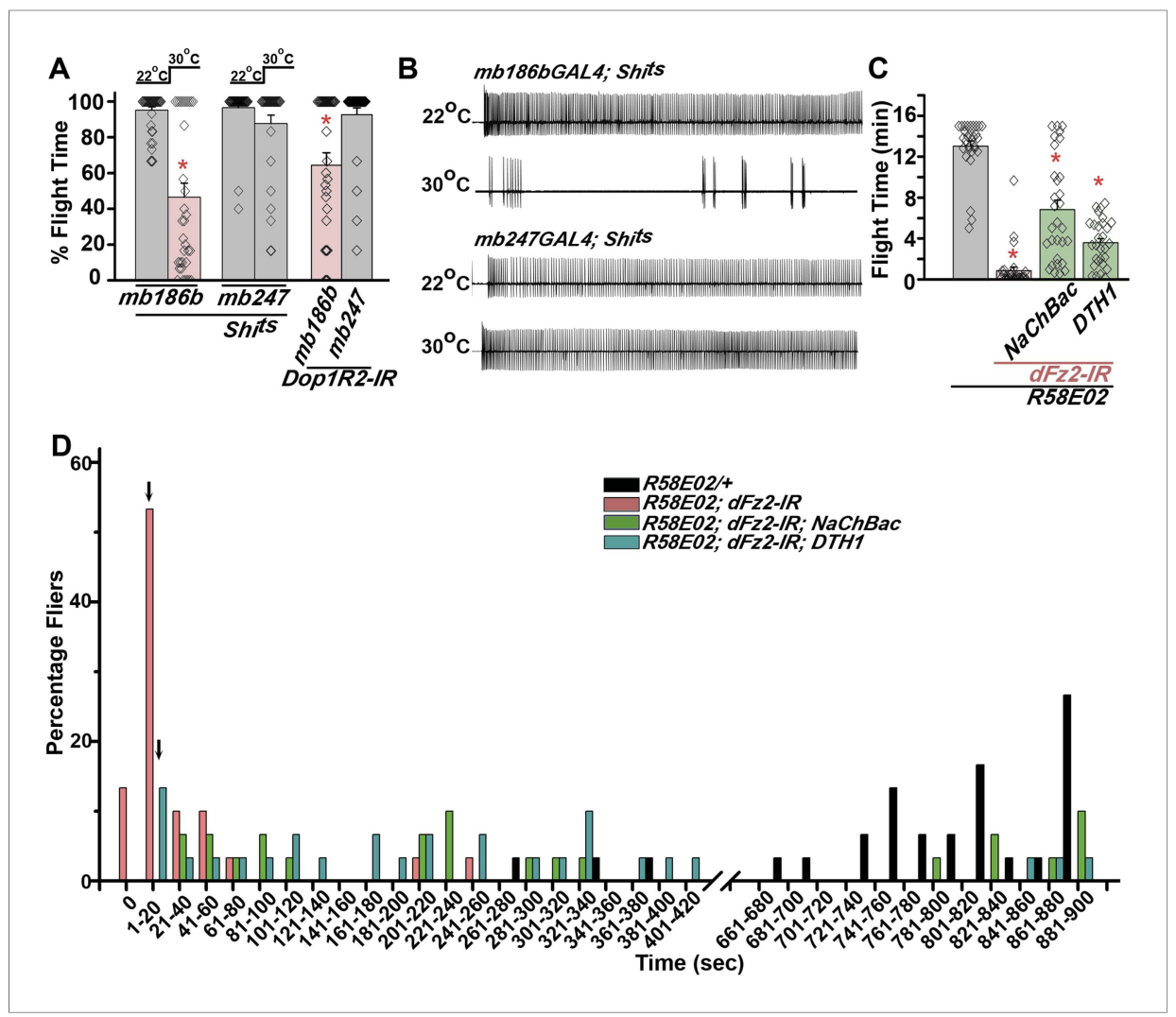

Figure 7. Mushroom body $\alpha^{\prime} / \beta^{\prime}$ neurons regulate flight through Dop1R2. (A) Percentage flight times of individual flies of the indicated genotypes. Flight defects were seen by reducing the activity of $\alpha^{\prime} / \beta^{\prime}$ neurons (mb186bGAL4, red bar) and by knockdown of Dop1R2 in mushroom body $\alpha^{\prime} / \beta^{\prime}$ neurons ( ${ }^{*} p<0$. 01, Mann-Whitney U-test).

(B) Electrophysiological responses from the DLMs showed similar responses as observed during flight. (C) Flight times during longer flight tests monitored over $15 \mathrm{~min}$ are shown. Over-expression of $\mathrm{NaChBac}$ rescued flight time partially when compared to knockdown to $d F z 2$ ( ${ }^{*} p<0.001$, Mann-Whitney U-test). (D) Percentage of flies that either do not initiate flight $(0 \mathrm{~s})$ or fly for time-periods within the binned intervals ( $20 \mathrm{~s}$ each) is shown for the indicated genotypes.

DOI: 10.7554/eLife.07046.028

The following figure supplements are available for figure 7 :

Figure supplement 1. Dopamine receptor knockdown in MB neurons.

DOI: 10.7554/eLife.07046.029

Figure supplement 2. Synaptic activity in $\alpha^{\prime} / \beta^{\prime}$ lobes required for flight. DOI: 10.7554/eLife.07046.030

Figure supplement 3. Knockdown of dFz2 does not affect climbing ability of flies. DOI: 10.7554/eLife.07046.031

Figure supplement 4. Maintenance of flight requires $\mathrm{dFz} / \mathrm{Ca}^{2+}$ signaling in dopaminergic neurons. DOI: 10.7554/eLife.07046.032

Figure supplement 5. Maintenance of flight requires $\mathrm{Fz} 2 / \mathrm{Ca}^{2+}$ signaling in dopaminergic neurons. DOI: 10.7554/eLife.07046.033

\section{Discussion}

\section{dFz2 signaling maintains TH levels in the dopaminergic PAM cluster}

Differentiation of neuronal subtypes, after genetic specification, is subject to multiple signals many of which generate and modify electrical activity of the cognate neurons (Borodinsky et al., 2014). We demonstrate a requirement for $\mathrm{dFz} 2 / \mathrm{Ca}^{2+}$ signaling for maintaining $\mathrm{TH}$ levels in a subset of central brain dopaminergic neurons-the PAM cluster. Our results support transcriptional regulation of $\mathrm{TH}$ and very likely the dopamine transporter (DAT) by $\mathrm{dFz} 2 / \mathrm{Ca}^{2+}$ signaling in the 
PAM neurons. A significant compensation of the flight deficit was observed in flies with $d F z 2$ knockdown in PAM neurons upon over-expression of the sodium channel $\mathrm{NaChBac}$, indicating that $\mathrm{dFz} 2 / \mathrm{Ca}^{2+}$ signaling also affects neural activity of PAM neurons. Moreover, flight deficits were observed upon expression of Shits in PAM neurons during the pupal stages, supporting a role for neural activity and synaptic transmission in their development. Increased TH transcripts and TH immunoreactivity after rescue by $\mathrm{NaChBac}$ suggests that $\mathrm{dFz} 2 / \mathrm{Ca}^{2+}$ signaling can in part be compensated by raised neural activity, and possibly the two signaling mechanisms function in parallel for maintaining TH transcription in PAM neurons. It is likely that in addition to TH and DAT, $\mathrm{dFz} 2 / \mathrm{Ca}^{2+}$ signals exert their influence on other transcripts in PAM neurons. Transcriptional profiling of the PAM neurons is necessary to address this possibility. Interestingly, despite an increase in the number of PAM neurons during pupal maturation (Figure 2-figure supplement 3), we do not observe an affect of $\mathrm{dFz} 2 / \mathrm{Ca}^{2+}$ signaling on the number of PAM neurons. Knockdown of dFz2 by DdcGAL4 that marks $>60$ PAM neurons resulted in flight time of $\sim 65 \%$ (Figure 1A), whereas a stronger flight deficit was observed with NP6510GAL4 which marks just 15 PAM neurons (Figure 2B). Moreover, the flight deficit obtained with R58E02GAL4 which marks 100 PAM neurons was similar to the flight deficit obtained with NP6510GAL4 (Figure 2B). Thus, the numbers of PAM neurons that express $d F z 2-I R$ do not correlate with the extent of observed flight deficits, suggesting that flight is regulated by a subset of PAM neurons and their projections to the MB. Further, analysis with GAL4 strains that mark PAM neuronal sub-domains would be helpful in identifying such flight specific PAM neurons.

Down-regulation of GFP fluorescence in PAMGAL4 strains upon dFz2 knockdown prevented direct analysis of their projections to the MB. Based on a recent study demonstrating similar PAM-MB connections for negative geotaxis (Riemensperger et al., 2013), we measured climbing in PAM > $d F z 2-I R$ flies. This appeared similar to controls (Figure 7-figure supplement 3), supporting the idea that connections of PAM neurons to the MB are maintained upon dFz2 knockdown. Thus as compared to climbing, flight appears more sensitive to the observed imbalance of $T H$. However, as expected inhibition of synaptic release from PAM neurons (R58E02GAL4>Shits; at $30^{\circ} \mathrm{C}$ ) affected both flight (Figure 4D) and climbing (Figure 7-figure supplement 3).

\section{dFz2 and calcium signaling in Drosophila}

In vertebrates, $\beta \gamma$ subunits of the trimeric $G_{\circ}$ protein activate phospholipase $C \beta$, which in turn enhances $\mathbb{I P}_{3}$ formation followed by $\mathbb{I P}_{3}$ receptor-mediated release of calcium from endoplasmic reticular stores (Rebecchi and Pentyala, 2000). In Xenopus embryos, non-canonical Wnt/Ca ${ }^{2+}$ signaling, acting through Fz receptors, activates the Nuclear Factor of Activated T cells (NFAT) which regulates transcription of genes required for dorsoventral axis formation (Saneyoshi et alo, 2002). Apart from NFAT, non-canonical $\mathrm{Fz} 2 / \mathrm{Ca}^{2+}$ signaling can also activate calcium calmodulindependent protein kinase $\|$ (CamKII) and protein kinase $C(P K C)$ which regulate activity of transcription factors, such as NFKB and CREB (Sheldahl et al., 1999; Kuhl et al., 2000; Slusarski and Pelegri, 2007). Non-canonical dFz2/ $\mathrm{Ca}^{2+}$ signaling has been poorly characterized in Drosophila. dFz2 can be cleaved and imported into the nucleus in Drosophila neurons (Mosca and Schwarz, 2010). However, we do not favor direct transcriptional control of $T H$ by cleaved dFz2 for the following reasons. Rescue of flight in dFz2 knockdown flies can be achieved by AcGo, itpr ${ }^{+}$, and dSTIM ${ }^{+}$ (Figure 2). These data support a link between $\mathrm{dFz} 2$ activation of $\mathrm{Go}$ at the membrane followed by intracellular $\mathrm{Ca}^{2+}$ release through the $\mathrm{IP}_{3} \mathrm{R}$ and $\mathrm{dSTIM}$-mediated calcium entry. This mechanism is broadly similar to what has been observed in vertebrates. Moreover, we did not detect nuclear dFz2 in PAM neurons (Figure 2-figure supplement 2).

Our observation that knockdown of $d F z 2$ reduced not only transcripts from the endogenous $\mathrm{TH}$ gene but also affected GFP expression from a DAT promoter transgene (R58E02GAL4>GFP) suggests co-ordinated transcriptional regulation of genes for maintenance of dopamine levels by $\mathrm{Fz} 2 / \mathrm{Ca}^{2+}$ signaling in PAM neurons. However, the molecular mechanism by which reduced $\mathrm{Fz} 2 / \mathrm{Ca}^{2+}$ signaling regulates transcription of $T H$ and very likely other genes, in PAM neurons remains to be elucidated.

\section{PAM-MB connectivity and flight}

Insect MBs are lobed structures located bilaterally in the protocerebrum of the central nervous system. Neuro-anatomical studies have demonstrated the presence of both efferent neurons arising 
from MB lobes as well as afferent connections supplying the MB lobes from protocerebral regions (Ito et al., 1998) including dopaminergic innervations from the PAM neurons (Mao and Davis, 2009). The Drosophila MB has been studied extensively as a central brain hub for olfactory associative memory and behavior (Heisenberg, 2003; Fiala, 2007). PAM-MB circuits are required for aversive as well as rewarding reinforcement of olfactory information (Aso et alı, 2012; Burke et al., 2012; Liu et al., 2012). Further analysis of our data revealed that majority ( 55\%) of flies with dFz2 knockdown in the PAM neurons fly for 1-20 s as compared to controls that can fly for 700-900 s or more. Rescue of flight deficits in flies with $d F z 2$ knockdown in PAM neurons either by over-expression of a sodium channel ( $\mathrm{NaChBac}$ ) or a transgene encoding TH (UAS-DTH1) supports a requirement for both neural activity and dopamine release from the PAM neurons for maintenance of longer flight bouts (Figure 7). Reduced flight times are very likely due to lack of dopaminergic reinforcement during flight arising from reduced strength of PAM-MB signaling.

The role of higher brain centres in Drosophila flight has been investigated primarily in the context of visual cues, and these studies identified the central complex as a key area for visual associative learning (Ofstad et alo, 2011). The flight circuit identified here appears similar to the one identified recently for the startle induced climbing response which requires PAM dopaminergic inputs to the $\beta^{\prime}$ lobe (Riemensperger et alo, 2013). Taken together, our findings support an emerging role for the Drosophila MB in coordinated motor behavior, previously considered unlikely (Wolf et alo, 1998). Dopaminergic inputs from the PAM to the MBs might help integrate olfactory sensory information with motor behavior essential in a natural environment. Further investigations should allow a better understanding of how MB centres for re-inforcement of olfactory memory interact with the flight motor system.

\section{Materials and methods}

\section{Fly rearing and stocks}

Drosophila was reared on corn flour/agar media supplemented with yeast, grown at $25^{\circ} \mathrm{C}$, unless otherwise mentioned in the experimental design. The pan-neuronal GAL4 driver (Elav ${ }^{C 155} G A L 4$ ), aminergic GAL4 (DdcGAL4) (Li et al., 2000) and mushroom body drivers c305aGAL4 and mb247GAL4 were obtained from Bloomington Stock Center, Bloomington, IN. mb186bGAL4 was obtained from Anja Beatrice Freidrich (MPG, Germany). The dopaminergic GAL4 (THGAL4), serotonergic GAL4 (TRHGAL4), and two other GAL4s, NP6510GAL4 and R58E02GAL4 were generously provided by Serge Birman (CNRS, ESPCl Paris Tech, France) (Riemensperger et al., 2013). The various dopaminergic subdomain GAL4 drivers used, THC'GAL4, THC1GAL4, and THF2GAL4, were obtained from Mark N Wu (Johns Hopkins University, Baltimore) (Liu et alo, 2012). The peptidergic GAL4 (P386GAL4) was obtained from Paul Taghert (Washington University, St. Louis) (Taghert et al., 2001).

UAS strains of Frizzled-2 RNAi (9739R-1(II), referred to as dFz2-IR in the text and figures) and itpr RNAi (1063-R2) were obtained from National Institute of Genetics Fly Stocks Centre, Kyoto, Japan (NIG). The UASRNAi strains for dSTIM (47073), dOrai (12221), Arrow (6707 and 36286), Dishevelled (101525), Shaggy (101538), DopECR (103494), Dop1R1 (107058), Dop1R2 (105324), Dop2R (11470, 11471), and TH (108879) were obtained from Vienna Drosophila RNAi center, Vienna, Austria (VDRC). UASRNAi strains for Frizzled2 (BL27568, BL31390 and BL31312) were also obtained from Bloomington Stock Center, Bloomington, IN. RNAi strains are referred to as IR indicating the presence of an interference RNA.

We obtained UAS-DTH1 from Serge Birman (CNRS, ESPCI Paris Tech, France), UASAcGo (GooQ205L) from Yu Fengwei (National University of Singapore, Singapore), UASAcGi (GiaQ205L) from Jurgen Knoblich (Institute of Molecular Biotechnology, Austria), UAS-PTX.16 from Gregg Roman (University of Houston, Texas) and UASFz2 from Stephen Cohen (Institute of Molecular Cell Biology, Singapore). AcGs (GasQ215L) BL6490; Go RNAi, BL34653; UASNaChBac, BL9468; UASDishevelled, BL9453 (Dsh); BL9522 (Dsh ${ }^{664 V}$ ), UASArmadilloactive, BL4782 (Arm ${ }^{\text {act }}$ ), and UAS-Shits (BL44222) were obtained from the Bloomington Stock Center, Bloomington, IN. UASdOrai+ (Venkiteswaran and Hasan, 2009), UASdSTIM+ (Agrawal et al., 2010), and UASAcGq3 (Ratnaparkhi et al., 2002) have been published. The GAL80 ${ }^{\text {ts }}$ strain with two inserts of tubP-GAL80 ${ }^{\text {ts }}$ on the second chromosome was generated by Albert Chiang, NCBS, Bangalore, India.

\section{Flight assay video and electrophysiological recordings}

Progeny were collected upon eclosion and aged for 3-4 days. For flight tests, flies were anaesthetized on ice for $15 \mathrm{~min}$ and a thin metal wire was glued between the neck and thorax region with the help of 
nail polish. To test air-puff-stimulated flight responses, videos were recorded for $30 \mathrm{~s}$ after a gentle mouth-blown air puff was delivered to the tethered fly. These videos were analyzed and percentage flight times were calculated. For short flight assays $30 \mathrm{~s}$ was taken as $100 \%$ flight time. For the long flight assay air-puff-stimulated flight times were monitored for $15 \mathrm{~min}$. For each genotype, a minimum of 30 flies were tethered and tested along with 30 control flies. Flight times of individual flies were noted, and data from a minimum of 30 flies were taken for calculation of the mean and standard error of mean (SEM). Significance testing between the raw data of control and experimental genotypes was performed with the Mann-Whitney U-test using GraphPad Prism 6 (GraphPad Software Inc, La Jolla, CA, USA). Data are represented as bar graphs of the mean percentage flight times. Diamonds inside each bar represent the flight time of individual flies.

Electrophysiological recordings were obtained from the indirect dorsal longitudinal flight muscles (DLMs) as described previously (Banerjee et al., 2004). Briefly, an un-insulated 0.127-mm tungsten electrode, sharpened by electrolysis to attain a $0.5 \mu \mathrm{m}$ tip diameter, was inserted in the DLMs (fiber a). A similar electrode was inserted in the abdomen for reference. Air-puff stimulated recordings were obtained for $30 \mathrm{~s}$. All recordings were performed using an ISO-DAM8A amplifier (World Precision Instruments, Sarasota, FL) with filter set up of $30 \mathrm{~Hz}$ (low pass) to $10 \mathrm{kHz}$ (high pass). Gap free mode of pClamp8 (Molecular Devices, Union City, CA) was used to digitize the data $(10 \mathrm{kHz})$ on a Pentium 5 computer equipped with Digidata 1322A (Molecular Devices). The duration of rhythmic action potential was analyzed using Clampfit (Molecular Devices) and the mean and standard error (SEM) were plotted using Origin 8.0 software (MicroCal, Origin Lab, Northampton, MA, USA). Spike durations in individual flies have been represented as diamonds within the histograms.

\section{Climbing assay}

Progeny were collected upon eclosion and aged for 3-4 days. To test for climbing, flies in batches of 10 were transferred into cylinder of diameter $2.5 \mathrm{~cm}$. Numbers of flies that crossed the $8 \mathrm{~cm}$ mark on the cylinder within $12 \mathrm{~s}$, after three gentle taps, were recorded. This procedure was repeated three times with three independent batches of flies. Means and SEM were calculated using the Origin 8.0 software (MicroCal, Origin Lab, Northampton, MA, USA).

\section{RNA isolation and cDNA synthesis}

For isolation of RNA, the central nervous system (CNS) was dissected from adult flies. For each genotype, three independent sets of RNA were isolated each from eight dissected CNS preparations. Total RNA was isolated using TRIzol Reagent (Invitrogen Life Technologies, Carlsbad, CA, USA) according to the manufacturer's specifications. Integrity of RNA was confirmed by visualization on a 1\% TAE (40 mM Tris pH 8.2, $40 \mathrm{mM}$ acetate, $1 \mathrm{mM}$ EDTA) agarose gel. Total RNA (500 ng) was treated with DNase in a volume of $45.5 \mu$ l with $1 \mu \mathrm{l}(1 \mathrm{U})$ DNase I (Amplification grade, Invitrogen Life Technologies, Carlsbad, CA, USA) with $1 \mathrm{mM}$ dithiothreitol (DTT) (Invitrogen Life Technologies, Carlsbad, CA, USA), 40U of RNase Inhibitor (Promega, Madison, WI, USA) in 5X First Strand Buffer (Invitrogen Life Technologies, Carlsbad, CA, USA) for $30 \mathrm{~min}$ at $37^{\circ} \mathrm{C}$ and heat inactivated for $10 \mathrm{~min}$ at $70^{\circ} \mathrm{C}$. The reverse transcription reaction was performed in a final volume of $50 \mu$ by addition of $1 \mu l$ (200U) Moloney murine leukemia virus (M-MLV) reverse transcriptase (Invitrogen Life Technologies, Carlsbad, CA, USA), $2.5 \mu \mathrm{l}(500 \mathrm{ng}$ ) random hexaprimers (MBI Fermentas, Glen Burnie, MD, USA) and $1 \mu$ of a $25 \mathrm{mM}$ dNTP mix (GE Healthcare, Buckinghamshire, UK). Samples were incubated for $10 \mathrm{~min}$ at $25^{\circ} \mathrm{C}$, then $60 \mathrm{~min}$ at $42^{\circ} \mathrm{C}$ and heat inactivated for $10 \mathrm{~min}$ at $70^{\circ} \mathrm{C}$. The polymerase chain reactions (PCRs) were performed using $1 \mu \mathrm{l}$ of cDNA as a template in a $25 \mu$ reaction under appropriate conditions to check the integrity of cDNA prepared.

\section{Quantitative PCR}

Real time quantitative PCR (qPCR) was performed on an ABI 7500 Fast machine (Applied Biosystems, Foster City, California, USA) operated with ABI 7500 software version 2 (Applied Biosystems, Foster City, California, USA) using MESA GREEN qPCR MasterMlx Plus for SYBR Assay I dTTp (Eurogentec, Belgium). Each qPCR experiment was repeated three times with independently isolated RNA samples. qPCRs were performed with rp49 primers as internal controls and primers specific to gene of interest using dilutions of 1:10. Sequences of the primers used in the $5^{\prime}$ to $3^{\prime}$ directions are given below. The sequence of the forward primer is given first in each case: $d f z 2$ GGTTACGGAGTGCCAGTCAT; CACAGGAAGAACTTGAGGTCC, rp49 CGGATCGATATGCTAAGCTGT; GCGCTTGTTCGATCCGTA, 
dsh CCAAATCCCAAGGGCTACTTC; ATAATACTGTCGTGCGATGTGAG sgg GCTGCTCGAGTA TACGCCC; CACTAGGCTGGGCTGTATTGA th GTTGCAGCAGCCCAAAAGAAC; GAGACCGTAATC ATTTGCCTTGC.

The cycling parameters were $95^{\circ} \mathrm{C}$ for $5 \mathrm{~min}, 40$ cycles of $95^{\circ} \mathrm{C}$ for $15 \mathrm{~s}$, and $60^{\circ} \mathrm{C}$ for $1 \mathrm{~min}$ followed by 1 cycle of $72^{\circ} \mathrm{C}$ for $5 \mathrm{~min}$. The fluorescent signal produced from the amplicon was acquired at the end of each polymerization step at $60^{\circ} \mathrm{C}$. A melt curve was performed after the assay to check for specificity of the reaction. The fold change of gene expression in the genotype relative to wild-type was determined by the comparative $\Delta \Delta$ Ct method (Lorentzos et al., 2003). In this method, the fold change $=2^{-\Delta \Delta \mathrm{Ct}}$ where $\Delta \Delta \mathrm{Ct}=\left(\mathrm{C}_{\mathrm{t}(\text { target gene })}-\mathrm{C}_{\mathrm{t}(\mathrm{rp} 49)}\right)_{\text {mutant2 }}-\left(\mathrm{C}_{\mathrm{t}(\text { target gene })}-\mathrm{C}_{\mathrm{t}(\mathrm{rp} 49)}\right)_{\text {Wild type }}$.

\section{Immunohistochemistry}

Immunohistochemistry was performed on Drosophila adult brains expressing cytosolic GFP (UASGFP) with the specified GAL4 strains, after fixing the dissected tissue in $4 \%$ paraformaldehyde. The following primary antibodies were used: mouse monoclonal anti-TH antibody (1:50, \#22941, ImmunoStar, Hudson, WI, USA), rabbit anti-GFP antibody (1:10,000; \#A6455, Molecular Probes, Eugene, OR, USA), mouse anti-Fz2 (1:20; \#12A7, DSHB, University of lowa). 12A7 was deposited to the DSHB by Nusse, R (DSHB Hybridoma Product 12A7). Fluorescent secondary antibodies were used at a dilution of 1:400 as follows: anti-rabbit Alexa Fluor 488 (\#A1108) and anti-mouse Alexa Fluor 568 (\#A1104, Molecular Probes, Eugene, OR, USA). After antibody staining, confocal analysis was performed on an Olympus Confocal FV1000 microscope and visualized using the FV10-ASW 1.3 viewer (Olympus Corporation, Tokyo, Japan).

\section{Data analysis}

Mean intensity of TH or GFP immunostaining was calculated using ImageJ Version 10.2 (U. S. National Institutes of Health, Bethesda, Maryland, USA, http://imagej.nih.gov/ij/, 1997-2014). Region of interest was drawn around each neuron and mean intensities were obtained for TH and GFP for all the neurons. Median shown by the horizontal line and spread of $25-75 \%$ of cell intensities represented as big square was calculated and plotted using Origin 8.0 software (MicroCal, Origin Lab, Northampton, MA, USA) with the data from all the neurons. Significant difference between the different groups of cell intensities was calculated using One-way analysis of variance (ANOVA) for $p<0.05$.

\section{Acknowledgements}

We thank Dr Krishnamurthy and NCBS Central Image and Flow Facility for help with the confocal imaging. Stocks obtained from the Bloomington Drosophila Stock Center (NIH P40OD018537) were used in this study.

\section{Additional information}

Funding

\begin{tabular}{lll} 
Funder & Grant reference & Author \\
\hline $\begin{array}{l}\text { National Centre for } \\
\text { Biological Sciences (NCBS) }\end{array}$ & Core funding & $\begin{array}{l}\text { Tarjani Agrawal, Gaiti } \\
\text { Hasan }\end{array}$ \\
$\begin{array}{lll}\text { National Institutes of Health } \\
\text { (NIH) }\end{array}$ & $\begin{array}{l}\text { Bloomington Drosophila } \\
\text { Stock Center P40OD018537 }\end{array}$ & Gaiti Hasan \\
\hline $\begin{array}{l}\text { Tata Institute of } \\
\text { Fundamental Research }\end{array}$ & Core funding & $\begin{array}{l}\text { Tarjani Agrawal, Gaiti } \\
\text { Hasan }\end{array}$ \\
\hline
\end{tabular}

The funders had no role in study design, data collection and interpretation, or the decision to submit the work for publication.

\section{Author contributions}

TA, Conception and design, Acquisition of data, Analysis and interpretation of data, Drafting or revising the article; $\mathrm{GH}$, Conception and design, Analysis and interpretation of data, Drafting or revising the article 


\section{References}

Agrawal N, Venkiteswaran G, Sadaf S, Padmanabhan N, Banerjee S, Hasan G. 2010. Inositol 1,4,5trisphosphate receptor and DSTIM function in Drosophila insulin-producing neurons regulates systemic intracellular calcium homeostasis and flight. The Journal of Neuroscience 30:1301-1313. doi: 10.1523/ JNEUROSCI.3668-09.2010.

Agrawal T, Sadaf S, Hasan G. 2013. A genetic RNAi screen for IP(3)/Ca(2)(+) coupled GPCRs in Drosophila identifies the PdfR as a regulator of insect flight. PLOS Genetics 9:e1003849. doi: 10.1371/journal.pgen.1003849.

Aso Y, Herb A, Ogueta M, Siwanowicz I, Templier T, Friedrich AB, Ito K, Scholz H, Tanimoto H. 2012. Three dopamine pathways induce aversive odor memories with different stability. PLOS Genetics 8:e1002768. doi: 10. 1371/journal.pgen.1002768.

Aso Y, Hattori D, Yu Y, Johnston RM, lyer NA, Ngo TT, Dionne H, Abbott LF, Axel R, Tanimoto H, Rubin GM. 2014. The neuronal architecture of the mushroom body provides a logic for associative learning. eLife 3:e04577. doi: 10.7554/eLife.04577.

Banerjee S, Lee J, Venkatesh K, Wu CF, Hasan G. 2004. Loss of flight and associated neuronal rhythmicity in inositol 1,4,5-trisphosphate receptor mutants of Drosophila. The Journal of Neuroscience 24:7869-7878. doi: 10. 1523/JNEUROSCI.0656-04.2004.

Borodinsky LN, Belgacem YH, Swapna I, Visina O, Balashova OA, Sequerra EB, Tu MK, Levin JB, Spencer KA, Castro PA, Hamilton AM, Shim S. 2015. Spatiotemporal integration of developmental cues in neural development. Developmental Neurobiology 75:349-359. doi: 10.1002/dneu.22254.

Brand AH, Perrimon N. 1993. Targeted gene expression as a means of altering cell fates and generating dominant phenotypes. Development 118:401-415.

Burke CJ, Huetteroth W, Owald D, Perisse E, Krashes MJ, Das G, Gohl D, Silies M, Certel S, Waddell S. 2012. Layered reward signalling through octopamine and dopamine in Drosophila. Nature 492:433-437. doi: 10.1038/ nature11614.

Ciani L, Boyle KA, Dickins E, Sahores M, Anane D, Lopes DM, Gibb AJ, Salinas PC. 2011. Wnt7a signaling promotes

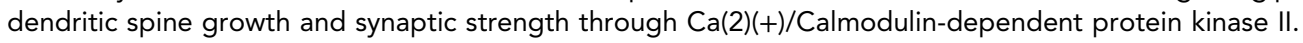
Proceedings of the National Academy of Sciences of USA 108:10732-10737. doi: 10.1073/pnas.1018132108.

Consoulas C, Restifo LL, Levine RB. 2002. Dendritic remodeling and growth of motoneurons during metamorphosis of Drosophila melanogaster. The Journal of Neuroscience 22:4906-4917.

Dietzl G, Chen D, Schnorrer F, Su KC, Barinova Y, Fellner M, Gasser B, Kinsey K, Oppel S, Scheiblauer S, Couto A, Marra V, Keleman K, Dickson BJ. 2007. A genome-wide transgenic RNAi library for conditional gene inactivation in Drosophila. Nature 448:151-156. doi: 10.1038/nature05954.

Fernandes J, VijayRaghavan K. 1993. The development of indirect flight muscle innervation in Drosophila melanogaster. Development 118:215-227.

Ferris J, Ge H, Liu L, Roman G. 2006. G(o) signaling is required for Drosophila associative learning. Nature Neuroscience 9:1036-1040. doi: 10.1038/nn1738.

Feske S, Gwack Y, Prakriya M, Srikanth S, Puppel SH, Tanasa B, Hogan PG, Lewis RS, Daly M, Rao A. 2006. A mutation in Orai1 causes immune deficiency by abrogating CRAC channel function. Nature 441:179-185. doi: $10.1038 /$ nature 04702 .

Fiala A. 2007. Olfaction and olfactory learning in Drosophila: recent progress. Current Opinion in Neurobiology 17: 720-726. doi: 10.1016/j.conb.2007.11.009.

Friggi-Grelin F, Iché M, Birman S. 2003. Tissue-specific developmental requirements of Drosophila tyrosine hydroxylase isoforms. Genesis 35:260-269. doi: 10.1002/gene.1082.

Friggi-Grelin F, Coulom H, Meller M, Gomez D, Hirsh J, Birman S. 2003. Targeted gene expression in Drosophila dopaminergic cells using regulatory sequences from tyrosine hydroxylase. Journal of Neurobiology 54:618-627. doi: 10.1002/neu.10185.

Gotz K. 1987. Course-control, metabolism and wing interference during ultralong tethered flight in Drosophila melanogaster. The Journal of Experimental Biology 128:35-46.

Heisenberg M. 2003. Mushroom body memoir: from maps to models. Nature Reviews. Neuroscience 4:266-275. doi: 10.1038/nrn1074.

Helfrich-Förster C, Wulf J, De Belle JS. 2002. Mushroom body influence on locomotor activity and circadian rhythms in Drosophila melanogaster. Journal of Neurogenetics 16:73-109.

Hopkins RS, Stamnes MA, Simon MI, Hurley JB. 1988. Cholera toxin and pertussis toxin substrates and endogenous ADP-ribosyltransferase activity in Drosophila melanogaster. Biochimica et Biophysica Acta 970: 355-362. doi: 10.1016/0167-4889(88)90135-8.

Ito K, Suzuki K, Estes P, Ramaswami M, Yamamoto D, Strausfeld NJ. 1998. The organization of extrinsic neurons and their implications in the functional roles of the mushroom bodies in Drosophila melanogaster Meigen. Learning \& Memory 5:52-77.

Kahsai L, Zars T. 2011. Learning and memory in Drosophila: behavior, genetics, and neural systems. International Review of Neurobiology 99:139-167. doi: 10.1016/B978-0-12-387003-2.00006-9.

Kitamoto T. 2001. Conditional modification of behavior in Drosophila by targeted expression of a temperaturesensitive shibire allele in defined neurons. Journal of Neurobiology 47:81-92. doi: 10.1002/neu.1018.

Krashes MJ, Keene AC, Leung B, Armstrong JD, Waddell S. 2007. Sequential use of mushroom body neuron subsets during drosophila odor memory processing. Neuron 53:103-115. doi: 10.1016/j. neuron.2006.11.021.

Kuhl M, Sheldahl LC, Park M, Miller JR, Moon RT. 2000. The Wnt/Ca2+ pathway: a new vertebrate Wnt signaling pathway takes shape. Trends in Genetics 16:279-283. doi: 10.1016/S0168-9525(00)02028-X. 
Li H, Chaney S, Roberts IJ, Forte M, Hirsh J. 2000. Ectopic G-protein expression in dopamine and serotonin neurons blocks cocaine sensitization in Drosophila melanogaster. Current Biology 10:211-214. doi: 10.1016/ S0960-9822(00)00340-7.

Liu C, Placais PY, Yamagata N, Pfeiffer BD, Aso Y, Friedrich AB, Siwanowicz I, Rubin GM, Preat T, Tanimoto H. 2012. A subset of dopamine neurons signals reward for odour memory in Drosophila. Nature 488:512-516. doi: 10.1038/nature11304.

Liu Q, Liu S, Kodama L, Driscoll MR, Wu MN. 2012. Two dopaminergic neurons signal to the dorsal fan-shaped body to promote wakefulness in Drosophila. Current Biology 22:2114-2123. doi: 10.1016/j.cub.2012.09.008.

Lorentzos P, Kaiser T, Kennerson ML, Nicholson GA. 2003. A rapid and definitive test for Charcot-Marie-Tooth 1A and hereditary neuropathy with liability to pressure palsies using multiplexed real-time PCR. Genetic Testing 7: 135-138. doi: 10.1089/109065703322146821.

Mao Z, Davis RL. 2009. Eight different types of dopaminergic neurons innervate the Drosophila mushroom body neuropil: anatomical and physiological heterogeneity. Frontiers in Neural Circuits 3:5. doi: 10.3389/neuro.04.005.2009.

McGuire SE, Le PT, Osborn AJ, Matsumoto K, Davis RL. 2003. Spatiotemporal rescue of memory dysfunction in Drosophila. Science 302:1765-1768. doi: 10.1126/science.1089035.

McGuire SE, Mao Z, Davis RL. 2004. Spatiotemporal gene expression targeting with the TARGET and gene-switch systems in Drosophila. Science's STKE 2004:pl6. doi: 10.1126/stke.2202004pl6.

Morel V, Arias AM. 2004. Armadillo/beta-catenin-dependent Wnt signalling is required for the polarisation of epidermal cells during dorsal closure in Drosophila. Development 131:3273-3283. doi: 10.1242/dev.01217.

Mosca TJ, Schwarz TL. 2010. The nuclear import of Frizzled2-C by Importins-beta11 and alpha2 promotes postsynaptic development. Nature Neuroscience 13:935-943. doi: 10.1038/nn.2593.

Nitabach MN, Wu Y, Sheeba V, Lemon WC, Strumbos J, Zelensky PK, White BH, Holmes TC. 2006. Electrical hyperexcitation of lateral ventral pacemaker neurons desynchronizes downstream circadian oscillators in the fly circadian circuit and induces multiple behavioral periods. The Journal of Neuroscience 26:479-489. doi: 10.1523/ JNEUROSCI.3915-05.2006.

Ofstad TA, Zuker CS, Reiser MB. 2011. Visual place learning in Drosophila melanogaster. Nature 474:204-207. doi: 10.1038/nature10131.

Pech U, Dipt S, Barth J, Singh P, Jauch M, Thum AS, Fiala A, Riemensperger T. 2013. Mushroom body miscellanea: transgenic Drosophila strains expressing anatomical and physiological sensor proteins in Kenyon cells. Frontiers in Neural Circuits 7:147. doi: 10.3389/fncir.2013.00147.

Pech U, Pooryasin A, Birman S, Fiala A. 2013. Localization of the contacts between Kenyon cells and aminergic neurons in the Drosophila melanogaster brain using SplitGFP reconstitution. The Journal of Comparative Neurology 521:3992-4026. doi: 10.1002/cne.23388.

Penton A, Wodarz A, Nusse R. 2002. A mutational analysis of dishevelled in Drosophila defines novel domains in the dishevelled protein as well as novel suppressing alleles of axin. Genetics 161:747-762.

Prakriya M, Feske S, Gwack Y, Srikanth S, Rao A, Hogan PG. 2006. Orai1 is an essential pore subunit of the CRAC channel. Nature 443:230-233. doi: 10.1038/nature05122.

Ratnaparkhi A, Banerjee S, Hasan G. 2002. Altered levels of Gq activity modulate axonal pathfinding in Drosophila. The Journal of Neuroscience 22:4499-4508.

Rebecchi MJ, Pentyala SN. 2000. Structure, function, and control of phosphoinositide-specific phospholipase C. Physiological Reviews 80:1291-1335.

Riemensperger T, Issa AR, Pech U, Coulom H, Nguyen MV, Cassar M, Jacquet M, Fiala A, Birman S. 2013. A single dopamine pathway underlies progressive locomotor deficits in a Drosophila model of Parkinson disease. Cell Reports 5:952-960. doi: 10.1016/j.celrep.2013.10.032.

Sadaf S, Hasan G. 2014. Serotonergic neurons of the Drosophila air-puff-stimulated flight circuit. Journal of Biosciences 39:575-583. doi: 10.1007/s12038-014-9449-5.

Sadaf S, Reddy OV, Sane SP, Hasan G. 2015. Neural control of wing coordination in flies. Current Biology 25: 80-86. doi: 10.1016/j.cub.2014.10.069.

Saneyoshi T, Kume S, Amasaki Y, Mikoshiba K. 2002. The Wnt/calcium pathway activates NF-AT and promotes ventral cell fate in Xenopus embryos. Nature 417:295-299. doi: 10.1038/417295a.

Serway CN, Kaufman RR, Strauss R, de Belle JS. 2009. Mushroom bodies enhance initial motor activity in Drosophila. Journal of Neurogenetics 23:173-184. doi: 10.1080/01677060802572895.

Sheldahl LC, Park M, Malbon CC, Moon RT. 1999. Protein kinase C is differentially stimulated by Wnt and Frizzled homologs in a G-protein-dependent manner. Current Biology 9:695-698. doi: 10.1016/S0960-9822(99)80310-8.

Sheldahl LC, Slusarski DC, Pandur P, Miller JR, Kuhl M, Moon RT. 2003. Dishevelled activates Ca2+ flux, PKC, and CamKII in vertebrate embryos. The Journal of Cell Biology 161:769-777. doi: 10.1083/jcb.200211094.

Slusarski DC, Pelegri F. 2007. Calcium signaling in vertebrate embryonic patterning and morphogenesis. Developmental Biology 307:1-13. doi: 10.1016/j.ydbio.2007.04.043.

Slusarski DC, Corces VG, Moon RT. 1997. Interaction of Wnt and a Frizzled homologue triggers G-protein-linked phosphatidylinositol signalling. Nature 390:410-413. doi: 10.1038/37138.

Spitzer NC. 2012. Activity-dependent neurotransmitter respecification. Nature Reviews. Neuroscience 13:94-106. doi: 10.1038/nrn3154.

Strausfeld NJ, Hirth F. 2013. Deep homology of arthropod central complex and vertebrate basal ganglia. Science 340:157-161. doi: 10.1126/science.1231828.

Strausfeld NJ, Sinakevitch I, Vilinsky I. 2003. The mushroom bodies of Drosophila melanogaster: an immunocytological and golgi study of Kenyon cell organization in the calyces and lobes. Microscopy Research and Technique 62:151-169. doi: 10.1002/jemt.10368. 
Strauss R. 2002. The central complex and the genetic dissection of locomotor behaviour. Current Opinion in Neurobiology 12:633-638. doi: 10.1016/S0959-4388(02)00385-9.

Taghert PH, Nitabach MN. 2012. Peptide neuromodulation in invertebrate model systems. Neuron 76:82-97. doi: 10.1016/j.neuron.2012.08.035.

Taghert PH, Hewes RS, Park JH, O'Brien MA, Han M, Peck ME. 2001. Multiple amidated neuropeptides are required for normal circadian locomotor rhythms in Drosophila. The Journal of Neuroscience 21:6673-6686.

van Amerongen R, Nusse R. 2009. Towards an integrated view of Wnt signaling in development. Development 136:3205-3214. doi: 10.1242/dev.033910.

Van Breugel F, Suver MP, Dickinson MH. 2014. Octopaminergic modulation of the visual flight speed regulator of Drosophila. The Journal of Experimental Biology 217:1737-1744. doi: 10.1242/jeb.098665.

Varela-Nallar L, Alfaro IE, Serrano FG, Parodi J, Inestrosa NC. 2010. Wingless-type family member 5A (Wnt-5a) stimulates synaptic differentiation and function of glutamatergic synapses. Proceedings of the National Academy of Sciences of USA 107:21164-21169. doi: 10.1073/pnas.1010011107.

Vecsey CG, Pirez N, Griffith LC. 2014. The Drosophila neuropeptides PDF and sNPF have opposing electrophysiological and molecular effects on central neurons. Journal of Neurophysiology 111:1033-1045. doi: 10.1152/jn.00712.2013.

Venkiteswaran G, Hasan G. 2009. Intracellular Ca2+ signaling and store-operated $\mathrm{Ca} 2+$ entry are required in Drosophila neurons for flight. Proceedings of the National Academy of Sciences of USA 106:10326-10331. doi: 10.1073/pnas.0902982106.

Vig M, Peinelt C, Beck A, Koomoa DL, Rabah D, Koblan-Huberson M, Kraft S, Turner H, Fleig A, Penner R, Kinet JP. 2006. CRACM1 is a plasma membrane protein essential for store-operated Ca2+ entry. Science 312:1220-1223. doi: 10.1126/science.1127883.

Vogt K, Schnaitmann C, Dylla KV, Knapek S, Aso Y, Rubin GM, Tanimoto H. 2014. Shared mushroom body circuits underlie visual and olfactory memories in Drosophila. eLife 3:e02395. doi: 10.7554/eLife.02395.

Waddell S. 2013. Reinforcement signalling in Drosophila; dopamine does it all after all. Current Opinion in Neurobiology 23:324-329. doi: 10.1016/j.conb.2013.01.005.

Wolf R, Wittig T, Liu L, Wustmann G, Eyding D, Heisenberg M. 1998. Drosophila mushroom bodies are dispensable for visual, tactile, and motor learning. Learning \& Memory 5:166-178.

Zars T. 2000. Behavioral functions of the insect mushroom bodies. Current Opinion in Neurobiology 10:790-795. doi: 10.1016/S0959-4388(00)00147-1. 